\title{
Oleate acid-stimulated HMMR expression by CEBPa is associated with nonalcoholic steatohepatitis and hepatocellular carcinoma
}

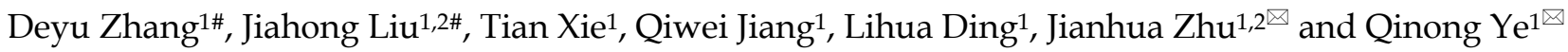 \\ 1. Department of Medical Molecular Biology, Beijing Institute of Biotechnology, Beijing 100850, China \\ 2. Department of Oncology, The Fourth Medical Center, PLA General Hospital, Beijing 100048, China. \\ \#These authors contributed equally to this work. \\ $\triangle$ Corresponding authors: Qinong Ye, Department of Medical Molecular Biology, Beijing Institute of Biotechnology, Beijing 100850, China. Phone: \\ 8610-66931830; Fax: 8610-88271216; E-mail: yeqn66@yahoo.com; Jianhua Zhu, Department of Oncology, The Fourth Medical Center, PLA General Hospital, \\ Beijing 100048, China. Phone: 8610-66848672; Fax: 8610-88455813; E-mail: zjhua168@sohu.com. \\ (c) The author(s). This is an open access article distributed under the terms of the Creative Commons Attribution License (https://creativecommons.org/licenses/by/4.0/). \\ See http://ivyspring.com/terms for full terms and conditions.
}

Received: 2020.06.22; Accepted: 2020.08.11; Published: 2020.08.27

\begin{abstract}
Non-alcoholic steatohepatitis (NASH) is a type of nonalcoholic fatty liver disease and has become a major risk factor for hepatocellular carcinoma (HCC). However, the underlying pathophysiological mechanisms are still elusive. Here, we identify hyaluronan-mediated motility receptor (HMMR) as a critical gene associated with $\mathrm{NASH} / \mathrm{HCC}$ by combination of bioinformatic analysis and functional experiments. Analysis of differentially expressed genes (DEGs) between normal controls and NASH/HCC identified 5 hub genes (HMMR, UBE2T, TYMS, PTTG1 and GINS2). Based on the common DEGs, analyses of univariate and multivariate Cox regression and the area under the curve (AUC) value of the receiver operating characteristic (ROC) indicate that HMMR is the most significant gene associated with NASH/HCC among five hub genes. Oleate acid (OA), one of fatty acids that induce cellular adipogenesis, stimulates HMMR expression via CCAAT/enhancer-binding protein $\alpha$ (CEBPa). CEBPa increases the expression of HMMR through binding to its promoter. HMMR promotes HCC cell proliferation in vitro via activation of $\mathrm{G} 1 / \mathrm{S}$ and $\mathrm{G} 2 / \mathrm{M}$ checkpoint transitions, concomitant with a marked increase of the positive cell cycle regulators, including cyclin DI, cyclin E, and cyclin B1. Knockdown of HMMR suppresses HCC tumor growth in nude mice. Our study identifies an important role of HMMR in NASH/HCC, and suggests that HMMR may be a useful target for therapy and prognostic prediction of $\mathrm{NASH} / \mathrm{HCC}$ patients.
\end{abstract}

Key words: non-alcoholic steatohepatitis, hepatocellular carcinoma, bioinformatics, oleate acid, cell cycle, CEBPa

\section{Introduction}

Non-alcoholic steatohepatitis (NASH) is a type of nonalcoholic fatty liver disease (NAFLD) characterized by hepatic triglyceride accumulation plus inflammation and hepatocyte injury [1]. According to reported data, NAFLD and NASH affect approximately $30 \%$ and $5 \%$ of the US population [2]. $\mathrm{NASH}$ is also the main chronic liver disease in the US and Europe, and is increasing worldwide [3]. There is mounting epidemiological evidence that it has become an emerging risk factor for hepatocellular carcinoma (HCC) [4]. Despite advances in HCC management, it is the second leading cause of cancer death in men and sixth in women worldwide [5]. The pathophysiological mechanisms linking NASH to HCC remain poorly understood, and are likely to be multifactorial. These may include dysregulated integrin and Hedgehog signaling, mitochondrial dysfunction, hepatic stellate cell (HSC) activation, and alteration of immune system [6].

Hyaluronan-mediated motility receptor (HMMR), also known as receptor for hyaluronatemediated motility (RHAMM), is a largely coiled-coil protein that can bind to microtubules and localize to the centrosome [7]. HMMR plays an important role in the regulation of spindle assembly in mitotic cells. HMMR expression is cell cycle-regulated with peak 
expression between late G2 phase and early mitosis. Expression of HMMR is upregulated in a variety of cancers, such as colorectal cancer [8], stomach cancer [9], endometrial cancer [10], prostate cancer [11], and multiple myeloma [12], and its high expression correlates with poor prognosis. In contrast, for some cancers, such as malignant peripheral nerve sheath tumors [13, 14] and seminomas [15], HMMR expression is downregulated and its low expression associates with poor patient survival. Very recently, HMMR was found to be one of the hub genes responsible for prognosis prediction of hepatocellular carcinoma (HCC) by bioinformatic analysis [16-19]. However, the clinical significance of HMMR linking normal liver to NASH to HCC and the biological function of HMMR in HCC remains unclear.

In this study, we aim to identify genes linking NASH and HCC through bioinformatics. We show that HMMR may be critical for the development of $\mathrm{NASH}$ and further progression to HCC. The accumulation of oleate acid, the most distinctive characteristic of liver tissues with NASH [20], increases HMMR expression through the transcription factor CCAAT enhancer binding protein a (CEBPa). Silencing of HMMR reduces HCC cell proliferation in vitro and tumor growth in vivo. Mechanistically, HMMR can regulate cell cycle progression in HCC cells.

\section{Materials and Methods}

\section{Plasmids, cell lines and reagents}

FLAG-tagged HMMR expression vector was constructed by inserting PCR amplified HMMR fragment into the pcDNA3 vector (Invitrogen) linked with FLAG tag at the amino terminus. The HMMR promoter luciferase reporters were made by inserting PCR-amplified HMMR promoter fragments into the pGL4-Basic vector (Promega). HepG2 and MHCC97H liver cancer cell lines were purchased from the American Type Culture Collection (Manassas, VA, USA). Small interfering RNAs (siRNAs) were synthesized by JTS scientific or GemmaPharma. The cDNA target sequences of siRNAs and/or short hairpin RNAs (shRNAs) for HMMR and CEBPa were listed in Table S1. Stable cell lines overexpressing HMMR shRNA were established by lentiviral transduction using pSIH-H1-Puro carrying HMMR shRNA. Anti-cyclin D1, anti-cyclin E, anti-cyclin B1 and anti-CEBPa were purchased from Santa Cruz Biotechnology; Anti-HMMR was from Proteintech.

\section{Data collection}

The GSE89632 and GSE126848 datasets were downloaded from the Gene Expression Omnibus (GEO, https://www.ncbi.nlm.nih.gov/geo/) database. The GSE89632 dataset contains gene expression profiles of 19 patients with NASH and 24 healthy controls [21]. The GSE126848 dataset contains gene expression profiles of 16 patients with NASH and 14 healthy controls [22]. In addition, mRNA expression profiles and clinical data from 366 HCC and 50 normal control samples were obtained from The Cancer Genome Atlas (TCGA, http:// cancergenome.nih.gov) database (TCGA-LIHC) [23]. The 50 normal control samples had matched tumor tissues.

\section{Identification of differentially expressed genes (DEGs) in NASH and HCC}

To investigate differentially expressed genes (DEGs) in each GEO dataset and TCGA-LIHC dataset, we used the limma $R$ package [24]. By controlling the false discovery rate (FDR), we define DEGs as genes with adj. $P$-value $<0.05$ and $\mid \log _{2}$ fold change (FC) $\mid>1$. The intersecting DEGs of NASH and HCC were used for further analysis.

\section{Functional and pathway enrichment analysis}

Functional and pathway enrichment analyses were performed for DEGs using the clusterProfiler $\mathrm{R}$ package [25], including Gene Ontology (GO) terms and Kyoto Encyclopedia of Genes and Genomes (KEGG) pathway enrichment analyses [26]. GO terms and KEGG pathways with an adj. $P$-value $<0.05$ were considered significant.

\section{Protein-protein interaction (PPI) network construction, module analysis and hub gene identification}

The PPI network was retrieved from the Search Tool for the Retrieval of Interacting Genes (STRING, http://www.string-db.org/). MCODE plugin in Cytoscape was used to identify the most significant module in the network based on the graph-theoretic clustering algorithm [27]. To identify hub genes in the PPI network, we use the cytoHubba plugin for Cytoscape [28]. Five analysis methods, namely Degree, Edge Percolated Component (EPC), Maximum Neighborhood Component (MNC), Density of Maximum Neighborhood Component (DMNC), and Maximal Clique Centrality (MCC), were used.

\section{Survival analysis of hub genes based on the TCGA-LIHC dataset}

After excluding HCC patients without survival data, the survival $R$ package was used to conduct survival analyses based on hub gene expression and overall survival (OS) in 365 HCC patients from TCGA-LIHC. Additionally, the online database Gene Expression Profiling Interactive Analysis (GEPIA) 
(http://gepia.cancer-pku.cn/) was used for diseasefree survival (DFS) analysis of hub genes in HCC patients from TCGA-LIHC. Log-rank $P$ value $<0.05$ is the threshold of statistical significance.

\section{HCC-specific prognostic model construction and assessment}

Univariate Cox proportional hazards regression analyses were used to identify individual common DEGs between NASH/HCC and adjacent normal tissues that affect the OS of $365 \mathrm{HCC}$ patients from the TCGA-LIHC dataset. Multivariate Cox regression analysis was used to establish a linear joint risk score of gene expression level (exp) using regression coefficient $\beta$. The risk score for each sample was calculated as follows: Risk score $=\sum \beta i \times \operatorname{expRNAi}[29]$. For survival analysis, the samples were divided into high- and low-risk groups based on the median or the best cutoff of risk scores. Subsequently, univariate and multivariate Cox regression analysis were performed to identify that the predictive effect of the prognostic model is independent of clinical factors. The prognostic model was assessed by the area under the curve (AUC) value of the receiver operating characteristic (ROC) curve, which was calculated using the survival ROC package of R. Finally, nomogram that integrates risk scores and other clinical factors was constructed to forecast the likelihood of 1-, 3-, 5-OS using the rms R package, and $P$ value $<0.05$ is the threshold of statistical significance.

\section{Cell culture and Oil Red $\mathbf{O}$ staining}

Cells were maintained in Dulbecco's modified Eagle's medium (DMEM) supplemented with $10 \%$ fetal bovine serum at $37{ }^{\circ} \mathrm{C}$ under a humidified atmosphere of $5 \%$ carbon dioxide. Cells were plated in 6-well plates at a density of $60 \%$ confluency. The next day, cells were treated with $25 \mu \mathrm{M} / 50 \mu \mathrm{M}$ oleate acid or DMSO (as a control), respectively. After treatment for $24 \mathrm{~h}$, cells were stained with Oil Red O and counterstained with hematoxylin [30].

\section{Transient Transfections}

For plasmid transfection, cells were seeded to $70-90 \%$ confluency at the time of transfection. The cells were transfected with the indicated plasmids using Vigofect according to the manufacturer's protocol (Vigorous Biotechnology). The transfected cells were collected after 24-48 h. For siRNA transfection, Lipofectamine RNAiMAX reagent was used according to the manufacturer's instructions (Invitrogen).

\section{Reverse-transcription quantitative real-time polymerase chain reaction (RT-qPCR)}

Total RNA was extracted from cultured cells and reverse-transcribed to cDNA using the RNeasy Mini kit (Qiagen) according to the manufacturer's protocol. Expression of mRNAs was determined by quantitative real-time polymerase chain reaction (qPCR) using SYBR Premix Ex Taq Master Mix (Takara). The relative expression was calculated by the comparative $\mathrm{Ct}$ method. The sequences of the primers used for RT-qPCR analysis are presented (Table S2).

\section{Cell proliferation and colony formation assays}

Cell proliferation was assessed by a CCK-8 Kit according to the manufacturer's instructions (Dojindo). For colony formation assay, transfected cells were seeded in 6-well plates at 3000 cells per well. Two weeks later, colonies were fixed with $4 \%$ paraformaldehyde and stained with $0.5 \%$ crystal violet for $30 \mathrm{~min}$. The number of colonies with diameters of more than $1.5 \mathrm{~mm}$ was counted.

\section{Luciferase reporter assay}

Cells seeded into 24-well plates were cotransfected with myc-CEBPa, the luciferase reporter with either wild-type or mutant HMMR promoter, and $\beta$-galactosidase reporter (internal control). Forty eight hours later, cells were harvested and analyzed for luciferase and $\beta$-galactosidase activities according to the manufacture's instruction (Promega). All transfection experiments were performed in triplicates and repeated 3 times.

\section{Chromatin immunoprecipitation}

ChIP assay was performed using the Magna ChIP Assay Kit (Millipore) according to the manufacturer's instructions. The collected DNA fragments were quantified by qPCR with listed primers (Table S2).

\section{Cell cycle analysis}

Cell cycle analysis was carried out using flow cytometry. Briefly, cells were fixed in $70 \%$ ethanol for more than $12 \mathrm{~h}$. After washing with PBS, fixed cells were incubated with RNase A $(0.2 \mathrm{mg} / \mathrm{mL})$ in PBS. Propidium iodide was then added to the cell suspension. Samples were analyzed by a FACSCalibur Flow Cytometer (Becton Dickinson).

\section{Statistical analysis}

All the experiments were performed in triplicate and repeated 3 times. Data are expressed as mean \pm standard deviation (SD), and were analyzed using SPSS 17.0 or R software. Statistical significance in cell line experiments was assessed by a two-tailed 
Student's $t$ test. $P<0.05$ was considered to be statistically significant.

\section{Results}

\section{Identification of commonly regulated differentially expressed genes between NASH/HCC and normal liver tissues}

A total of 50 paired HCC and adjacent normal tissues from the TCGA-LIHC dataset were analysed. The clinicopathological features were shown in Table S3. In this dataset, 1530 upregulated and 1863 downregulated differentially expressed genes (DEGs) were identified in HCC tissues compared with non-tumor tissues. In the GSE89632 dataset, 135 upregulated and 161 downregulated DEGs were identified in NASH patients compared with healthy controls. In the dataset GSE126848, 715 upregulated and 527 downregulated DEGs were identified in NASH patients compared with healthy controls. The volcano plot illustrating the gene expression profile of this dataset was shown in Figure 1A. There were 12 common upregulated DEGs and 10 common downregulated DEGs between NASH/HCC and adjacent normal tissues (Figure 1B). Seven genes out of the 22 common DEGs, such as FABP4, AKR1B10, and UBD, have been demonstrated to be the critical genes related to both NASH and HCC [31-36]. Some of the other 15 genes have been reported to be associated with only NASH or HCC. In addition, the heatmap illustrated the expression of common DEGs in HCC tissues or non-tumor tissues from the TCGA-LIHC (Figure 1C). Another heatmap illustrated the expression of common DEGs in NASH tissues or normal liver tissues (Figure 1D).

\section{PPI network construction and hub gene identification}

A PPI network for 22 common DEGs was built by the STRING online database, and 20 most credible direct interactors were joined in analysis (Figure 2A). The network shows the interconnectedness of 22 common DEGs between NASH/HCC, with upregulated ones being red and downregulated ones being green. There were several PPI prediction clusters analyzed by MCODE plugin in Cytoscape. Finally, we extracted the most significant module, which included five upregulated DEGs and 15 interactors (Figure 2B). These five upregulated DEGs also were identified as top five hub genes among DEGs by algorithms, MCC and DMMC (Table S4). Thus, the five genes, namely HMMR, ubiquitin conjugating enzyme E2 T (UBE2T), thymidylate synthetase (TYMS), pituitary tumor transforming gene 1 (PTTG1), and GINS complex subunit 2 (GINS2), were chosen for further analysis.

\section{Functional and pathway enrichment analysis}

For 22 common DEGs and the top 20 direct interactors, GO terms showed that changes in biological process included significant enrichment of DNA replication, mitosis associated catabolic process, etc. (Figure 2C). Changes in molecular function (MF) included significant enrichment of DNA helicase activity, DNA replication origin binding, ribonucleotide binding, single-stranded DNA-dependent ATPase activity, etc. Changes in cellular component (CC) included significant enrichment of genes related to the cell nucleus. KEGG pathway enrichment analysis demonstrated that cell cycle, meiosis, DNA replication and folate biosynthesis were significantly enriched (Figure 2D). The enrichment of cell cycle was consistent with previously reported researches on the five hub genes. HMMR was shown to bind to microtubule during mitotic spindle formation, suggesting its function in cell division [37]. UBE2T knockdown induces G1/S cell cycle arrest in HCC cells [38]. Silencing of PTTG1 inhibits cell proliferation and inhibits cyclin D1 expression in cholangiocarcinoma cells [39]. TYMS is an essential rate-limiting enzyme in the nucleotide metabolism, and is involved in DNA synthesis [40]. GINS2 knockdown also inhibits cell viability and induces cell cycle arrest in pancreatic cancer cells [41]. Thus, abnormal cell cycle may be the intrinsic related mechanism of NASH and HCC. Taken together, both GO terms and KEGG analysis indicated that some common DEGs might play an important role in cell cycle regulation in HCC cells and might have a role for NASH to HCC.

\section{The validation of five hub genes in TCGA-LIHC dataset}

We further explored associations between the hub gene expression and clinical stage in 343 HCC patients with clinical information from TCGA-LIHC dataset. The mRNA expression levels of HMMR, UBE2T, TYMS, PTTG1 and GINS2 significantly increased in those with advanced clinical stage (Figure 3A). Notably, the expression levels decreased in stage IV, which might be due to the small sample size. Moreover, high mRNA expression of the five hub genes, especially HMMR, was significantly associated with shorter overall survival (OS) in 365 HCC patients with survival information from TCGA-LIHC dataset (Figure 3B). High mRNA expression of the five hub genes was also correlated with shorter disease free survival (DFS) (Figure S1). 
A
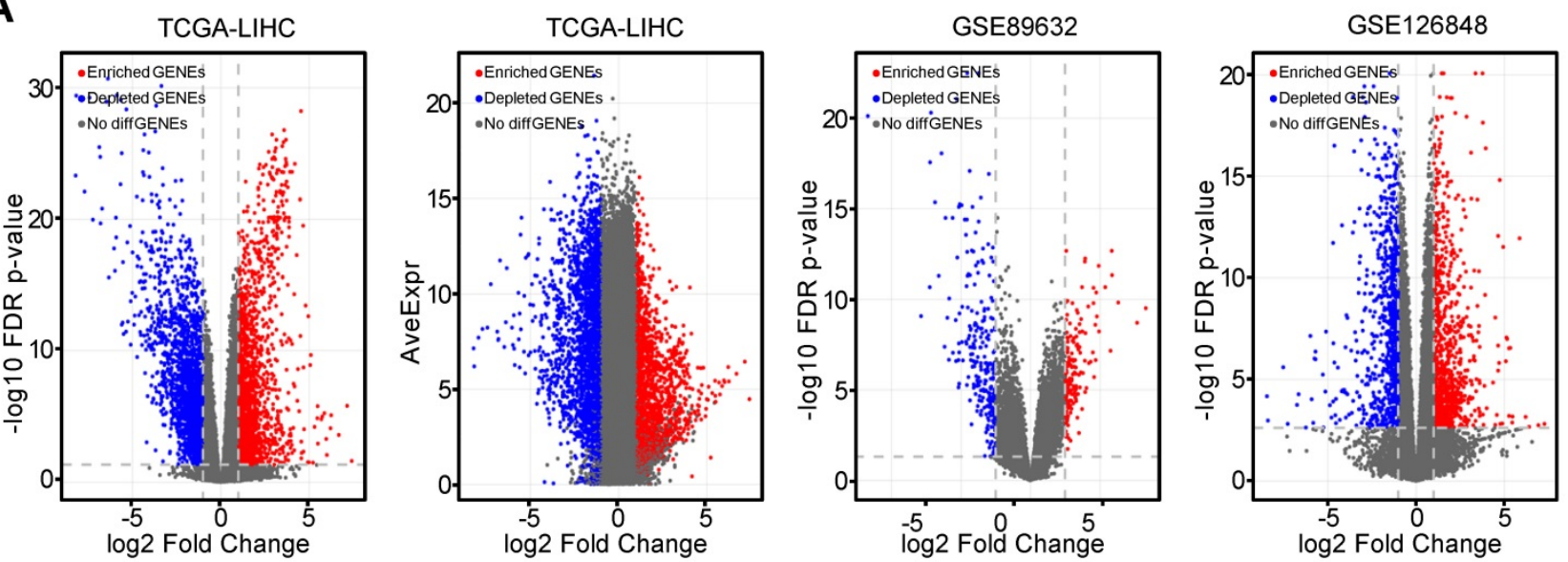

B
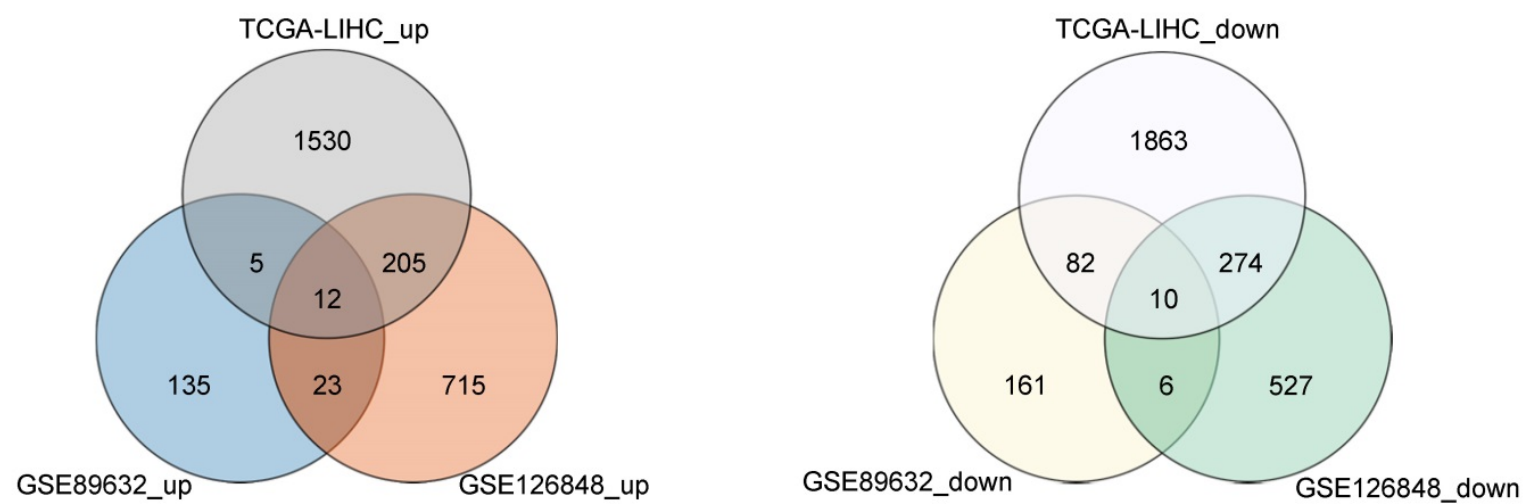

C

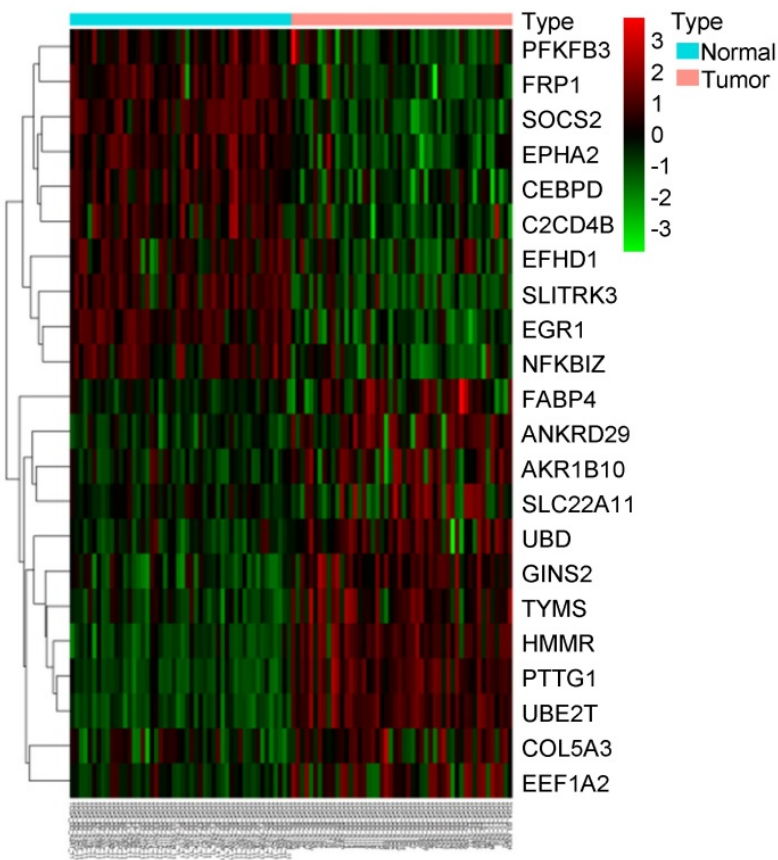

D

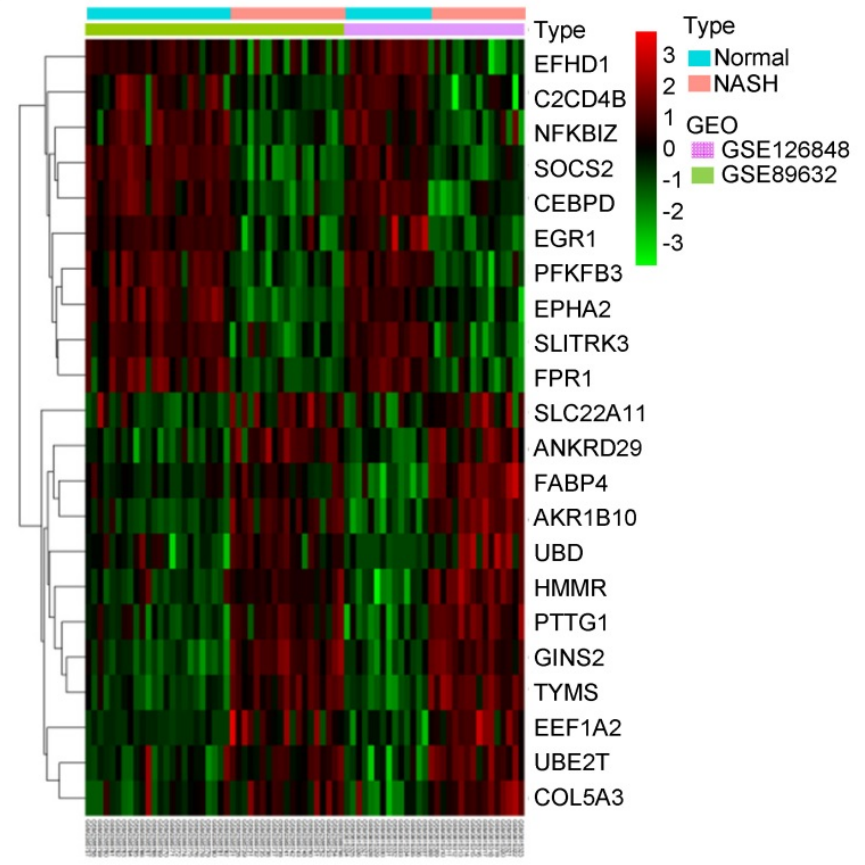

Figure 1. The common differentially expressed genes (DEGs) between normal controls and NASH/HCC. (A) The volcano plot showing DEGs in 366 HCC samples from TCGA-LIHC dataset, 43 samples from GSE89632 and 30 samples from GSE126848 dataset, respectively. (B) The common differentially up/down-expressed genes based on GSE89632, GSE126848 and TCGA-LIHC datasets. (C and D) The heatmaps illustrating the expression of common DEGs based on TCGA-LIHC (C), GSE89632 and GSE126848 (D) datasets. 
A

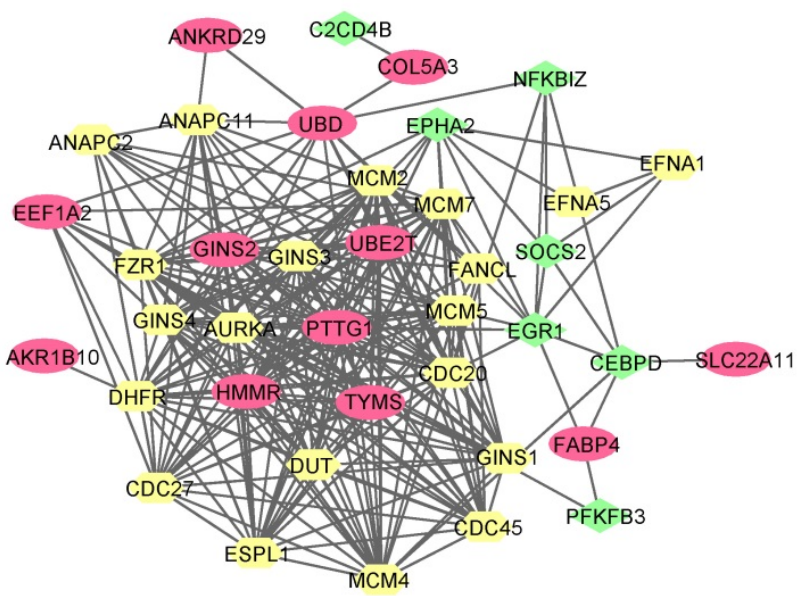

B

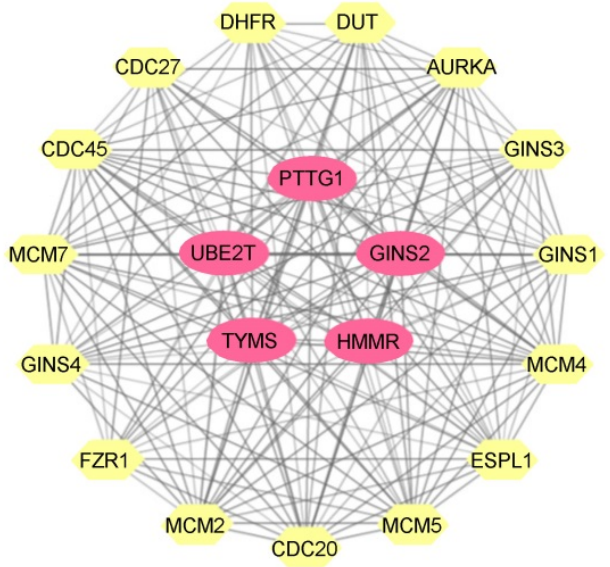

C

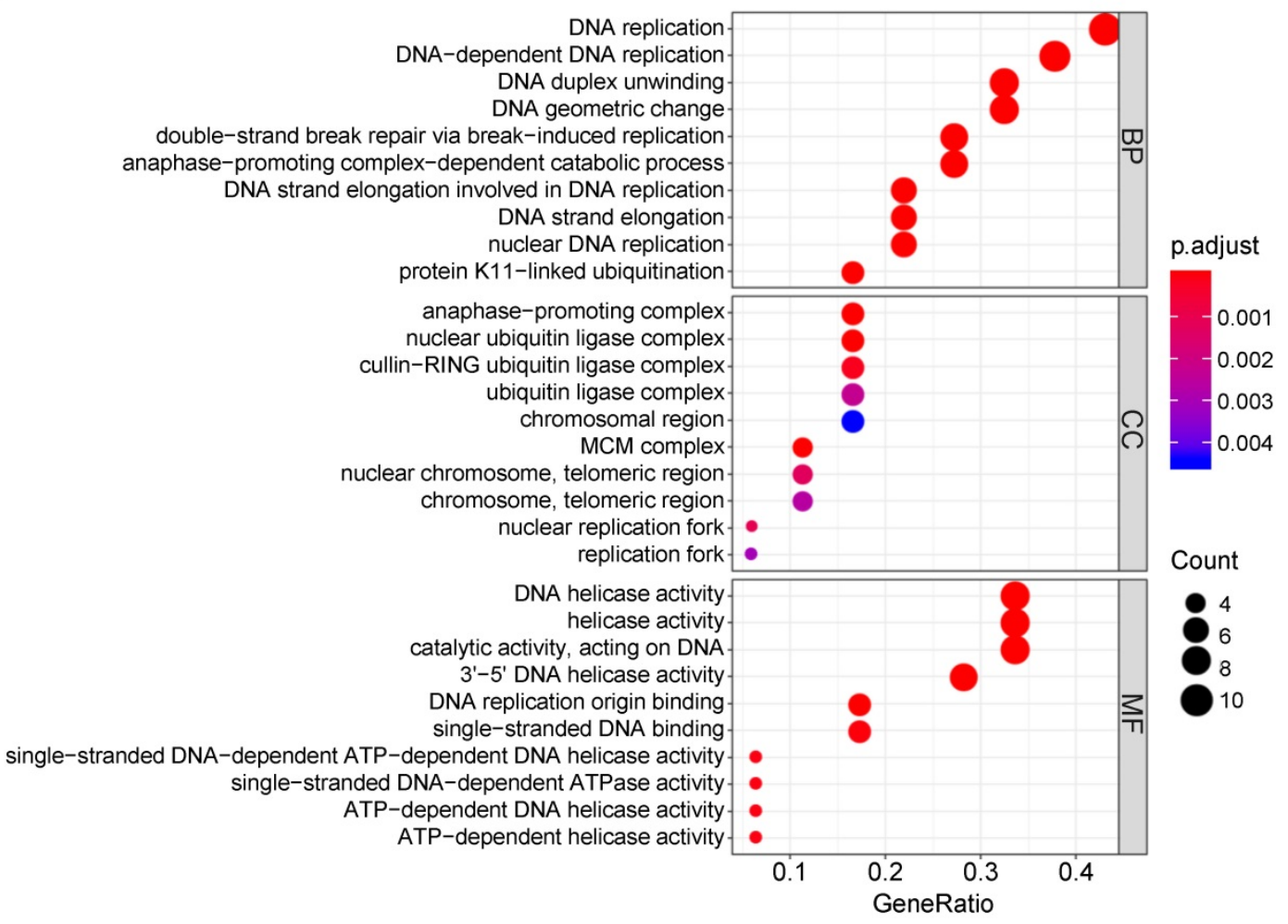

D

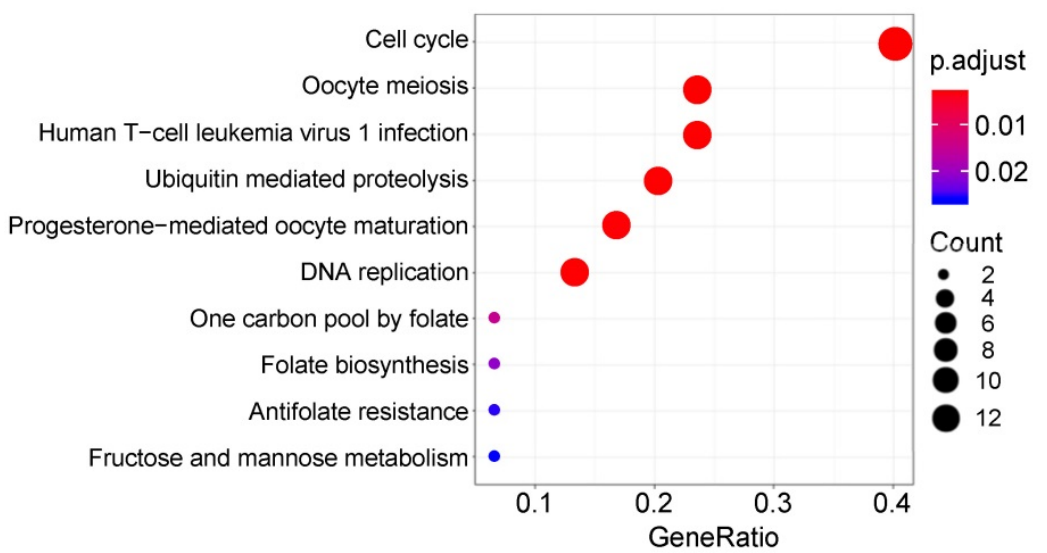

Figure 2. Functional enrichment analysis based on the DEGs. (A) Entire PPI network analysis of the DEGs and their direct interactors. (B) Identification of the most significant module and hub genes of the DEGs. (C) The top 10 changes in BP/MF/CC of GO analysis based on the DEGs and their direct interactors. (D) Top 10 enriched KEGG pathways based on the DEGs and their direct interactors. 
A
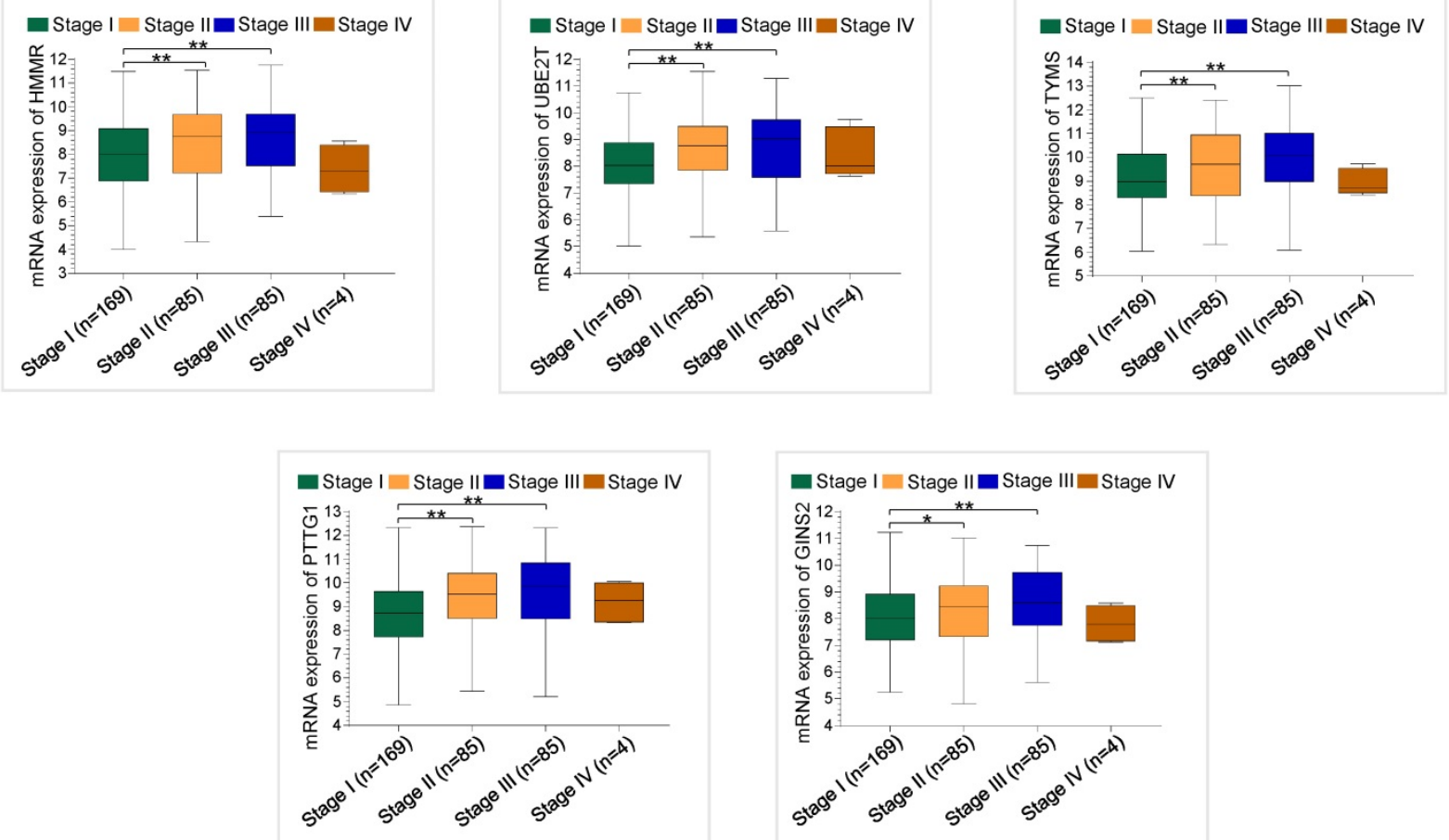

B
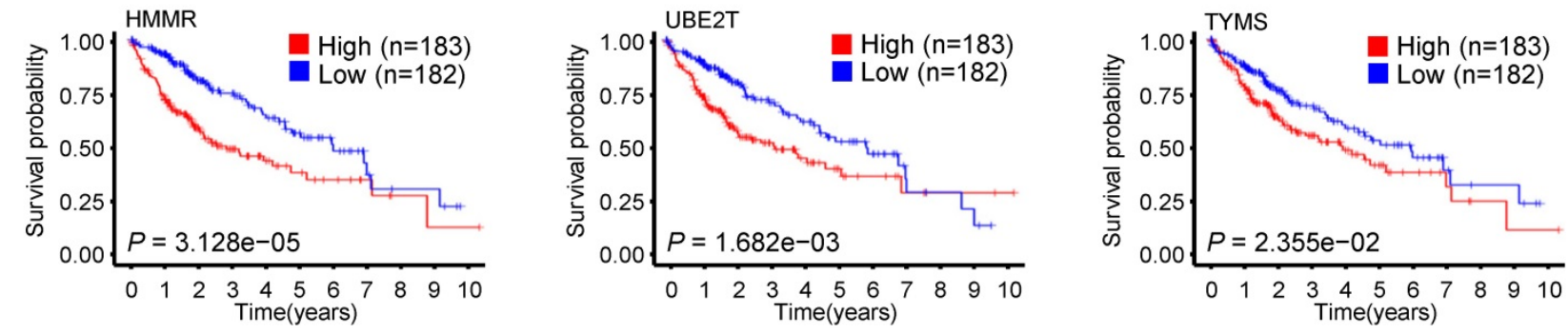

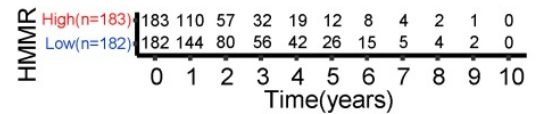

$\vdash$ High(n=183) $\begin{array}{lllllllllll}183 & 114 & 59 & 36 & 23 & 13 & 9 & 5 & 3 & 2 & 0\end{array}$

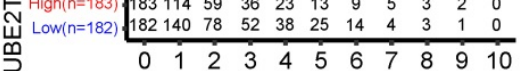

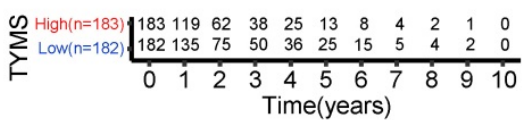
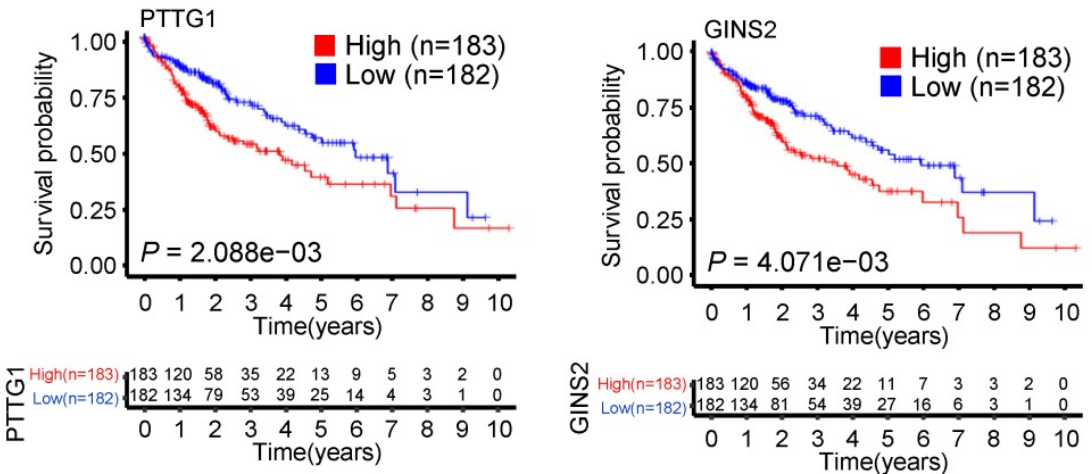

Figure 3. The validation of clinical significance of five hub gene expression in HCC patients from TCGA-LIHC dataset. (A) The relationship between five hub gene mRNA expression and clinical stages in $343 \mathrm{HCC}$ patients from TCGA-LIHC dataset $(* P<0.05$ versus stage $\mathrm{I}$, **P $<0.01$ versus stage $\mathrm{I})$. (B) The association between five hub gene expression and overall survival in $365 \mathrm{HCC}$ patients from TCGA-LIHC dataset.

\section{Identification of prognostic signature}

To determine the association between common
DEGs and patients' outcomes, 22 common DEGs were firstly submitted to univariate Cox proportional hazards regression. Eight genes, involving all five hub 
genes, were identified to have a significant prognostic value (Figure 4A). Then, multivariate Cox proportional hazards regression analysis screened out four genes: HMMR, aldo-keto reductase family 1 member B10 (AKR1B10), 6-phosphofructo-2kinase/fructose-2,6-bisphosphatases (PFKFB3), and suppressor of cytokine signaling 2 (SOCS2) (Figure 4B). Four-mRNA based prognostic signature was constructed and the risk-score formula used to calculate the risk score was as follows: $\left(0.276^{*} \mathrm{HMMR}\right.$ $+0.179 *$ AKR1B10 + 0.126*PFKFB3 - 0.495*SOCS2). The concordance index of this prognostic model was 0.692 , indicating a certain predictive effect. According to the median value of the prognostic risk score, 365 HCC patients from the TCGA-LIHC dataset were divided into low- and high-risk groups. The distribution of the risk score along with the corresponding OS data and the expression level of three genes in the prognostic model were plotted (Figure 4C). As depicted in the picture, patients with higher risk scores tended to experience a shorter OS time and higher death rate.

\section{Prognostic signature validation and nomogram construction}

We conducted the univariate Cox proportional hazards regression analysis to screen significant clinical features for prognosis, including age, gender, body mass index (BMI, weight in kilograms divided by the square of height in metres), grade, pathologic stage and the risk score of prognostic model were included. Only pathologic stage and the risk score of prognostic model had an effect on prognosis of HCC patients (Figure 4D). To determine whether the 4-mRNA-based prognostic signature is independent prognostic factor for HCC patients, the multivariate Cox proportional hazards regression analysis was performed using risk score and other clinical features. Pathologic stage and risk score maintained a significant and independent factor for prognosis prediction $(P<0.001)$ (Figure 4E). Additionally, the area under the curve (AUC) value of prognostic model was $0.761,0.697$ and 0.708 in 1-, 3-, 5-year survival prediction, respectively (Figure 4F). According to 1-year survival prediction, the AUC value of prognostic model was superior to other clinical factors. The AUC value of HMMR alone was 0.725 , and was an alternative to the prediction model with the four genes, HMMR, AKR1B10, PFKFB3, and SOCS2 (0.761). The comprehensive nomogram was constructed for individualized prediction of 1-, 3-, and 5 -year OS that intergrated prognostic features (age, gender, BMI, grade, pathologic stage and risk score) (Figure 4G).

\section{Oleate acid elevates the expression of HMMR via CEBPa}

As the AUC value of ROC analysis for the hub gene HMMR alone is similar to that of 4-mRNA-based prognostic signature, we explored the mechanism of elevated HMMR expression in NASH and HCC. $\mathrm{NASH}$ is characterized by accumulation of fat in liver cells. Oleate acid (OA), one of fatty acids that induce cellular adipogenesis, can promote the proliferation of HCC cells $[42,43]$. OA-induced lipid accumulation in HepG2 liver cancer cells is a well-established model for the investigation of hepatic steatosis [20]. As expected, Oil Red O staining showed that HepG2 cells treated with OA exhibited elevated intracellular lipid storage compared to the control cells (Figure 5A). Consistent with previous report (25), OA reduced the expression of phosphatase and tensin homolog (PTEN) (Figure 5B), a vital tumor suppressor gene in hepatocellular carcinoma. Importantly, OA markedly stimulated HMMR expression at the transcriptional and translational levels.

To investigate the mechanism underlying OA-induced HMMR expression, we predicted transcription factors based on the HMMR promoter by TFBIND. Among them, CEBPa is a key transcription factor associated with lipid metabolism, and was reported to be induced by OA [44]. Indeed, CEBPa overexpression increased HMMR mRNA and protein expression in HepG2 and MHCC-97H liver cancer cells, and OA further promoted CEBPastimulated HMMR expression (Figure 5C). As a control, CEBP $\beta$ did not alter the expression of HMMR. In contrast, in HepG2 and MHCC-97H cells, knockdown of CEBPa reduced HMMR mRNA and protein expression, and almost abrogated OAinduced HMMR expression (Figure 5D), indicating CEBPa-dependent HMMR induction by OA. ChIP assay showed that CEBPa was recruited to a region approximately 700-bp upstream of HMMR transcriptional start site, but not the other regions (Figure 5E). Luciferase reporter assays demonstrated that CEBPa increased the activity of wild-type HMMR promoter reporter containing putative CEBPa binding site, but not the reporter in which the putative binding site for CEBPa was mutated, in HepG2 and MHCC-97H cells (Figure 5F). These data suggest that CEBPa promotes HMMR gene transcription through binding to its promoter.

\section{HMMR promotes HCC cell proliferation}

Next, we explored whether HMMR plays a role in HCC cell proliferation. We first investigated the effect of HMMR overexpression on anchoragedependent growth of HCC cells. HepG2 cells transfected with FLAG-tagged HMMR grew much 
faster than those transfected with empty vector (Figure 6A). Moreover, colony formation assays revealed that colony number and colony size were larger in HMMR-overexpressing HepG2 cells than those in empty vector-containing cells. In contrast, HepG2 cells transfected with HMMR shRNA grew more slowly than those transfected with control shRNA (Figure 6B). Reexpression of HMMR in the HMMR knockdown cells rescued this effect. Knockdown of HMMR with HMMR shRNA in HepG2 cells decreased the colony number and size. Again, reexpression of HMMR in the HMMR knockdown cells rescued this effect. Similar results were obtained in MHCC-97H cells infected with HMMR-expressing or HMMR shRNA plasmids (Figure S2A and S2B). These results reveal that HMMR increases the proliferation and colony formation of HCC cells.

\section{HMMR activates the $G 1 / S$ and $G 2 / M$ transitions in HCC cells}

To elucidate the mechanism by which HMMR promotes HCC cell proliferation, we analyzed enriched pathways by GSEA with 366 HCC samples from the TCGA-LIHC dataset. Interesting, the cell cycle pathway was significantly enriched (Figure 6C). Hence, we investigated the effect of HMMR on cell cycle distribution by flow cytometry analysis. Compared with the control cells, overexpression of HMMR in HepG2 cells resulted in a reduction in the proportion of cells in G0/G1 phase (from $48.54 \%$ to $43.76 \%$ ) and $\mathrm{G} 2 / \mathrm{M}$ phase (from $21.35 \%$ to $16.97 \%$ ) but an increase in the proportion of cells in $S$ phase (from $30.11 \%$ to $39.27 \%$ ) (Figure 6D). In contrast, knockdown of HMMR in HepG2 cells significantly increased the proportion of cells in both G0/G1 (48.76\% to $54.03 \%$ ) and G2/M phase (from $21.47 \%$ to $24.89 \%)$, accompanied by decreased proportion of cells in S phase $(29.77 \%$ to $21.08 \%$ ) (Figure 6E). Reexpression of HMMR in the knockdown cells recued these effects. Similar results were obtained in MHCC-97H cells (Figure S2C and S2D). These data suggest that HMMR activates both the G1/S and the $\mathrm{G} 2 / \mathrm{M}$ transitions in HCC cells.

\section{HMMR regulates the expression of $G 1$ and $G 2$ phase-related proteins in HCC cells}

Since HMMR regulates cell cycle distribution, we examined the expression of several important cell cycle-related proteins in HMMR knockdown or overexpressing HCC cells. Overexpression of HMMR in HepG2 cells and MHCC-97H cells increased the expression of the G1/S-phase markers cyclin D1 and cyclin E, as well as the G2/M-phase marker cyclin B1 (Figure 6F and Figure S2E). However, the expression of cyclin A and the cell cycle inhibitor p21 was not changed in HMMR- overexpressing cells. On the contrary, knockdown of HMMR in HepG2 cells and MHCC-97H decreased the expression of cyclin D1, cyclin E and cyclin B1 (Figure 6G and Figure S2F). Reexpression of HMMR in the HMMR knockdown cells rescued this effect. Consistent with the HMMR overexpression results, HMMR knockdown did not alter the expression of cyclin A and p21.

\section{Knockdown of HMMR suppresses HCC tumor growth in nude mice}

Next, the effect of HMMR knockdown on HCC tumor growth in nude mice was investigated. HepG2 cells stably infected with HMMR shRNA lentivirus or empty vector were injected subcutaneously in the dorsal of each nude mouse. Compared with the control groups, knockdown of HMMR significantly suppressed HCC tumor growth in nude mouse (Figure 7A). As expected, the HepG2 tumors in mice inoculated with HMMR shRNA showed decreased expression of HMMR, cyclin D1, cyclin E, and cyclin B1 (Figure 7B).

\section{Discussion}

In this study, 12 common upregulated and 10 common downregulated DEGs are identified in $\mathrm{NASH}$ and HCC when compared with normal controls. Five genes, including HMMR, UBE2T, TYMS, PTTG1 and GINS2 are identified as hub genes. All five hub genes are found to be independent adverse prognostic biomarkers for OS and DFS. Univariate and multivariate Cox regression analysis and prognostic model analysis reveal that HMMR is the most significant gene associated with $\mathrm{NASH} / \mathrm{HCC}$ among five hub genes. Furthermore, HMMR promotes HCC tumor growth by activation of cell cycle progression, accompanied by changes in expression of cell cycle regulators (Figure 7C). These findings indicate that HMMR may play an important role in the development of NASH and further progression to HCC.

NASH has become a major carcinogenic factor for HCC, due to the increase in obesity worldwide [1, 45]. There is growing literature suggesting that various aspects contribute to the development of this prevalent and serious chronic disease, such as molecular events, immune status, biochemical reaction, and genetic function [46-48]. However, the pathophysiologic development of NASH and subsequent progression to HCC is still largely unclear [49]. Our study identifies genetic and molecular events, which may be involved in the process from normal liver to NASH to HCC through bioinformatic analysis. We used GEO datasets for NASH and 
TCGA-LIHA datasets for HCC to screen for hub genes which transform normal liver to NASH to HCC. We found that HMMR was overexpressed in NASH patients compared to health controls, and negatively correlated with poor prognosis in HCC patients, suggesting that HMMR may be a potential monitoring target for prediction of NASH or HCC patients' progression and prognosis.
A

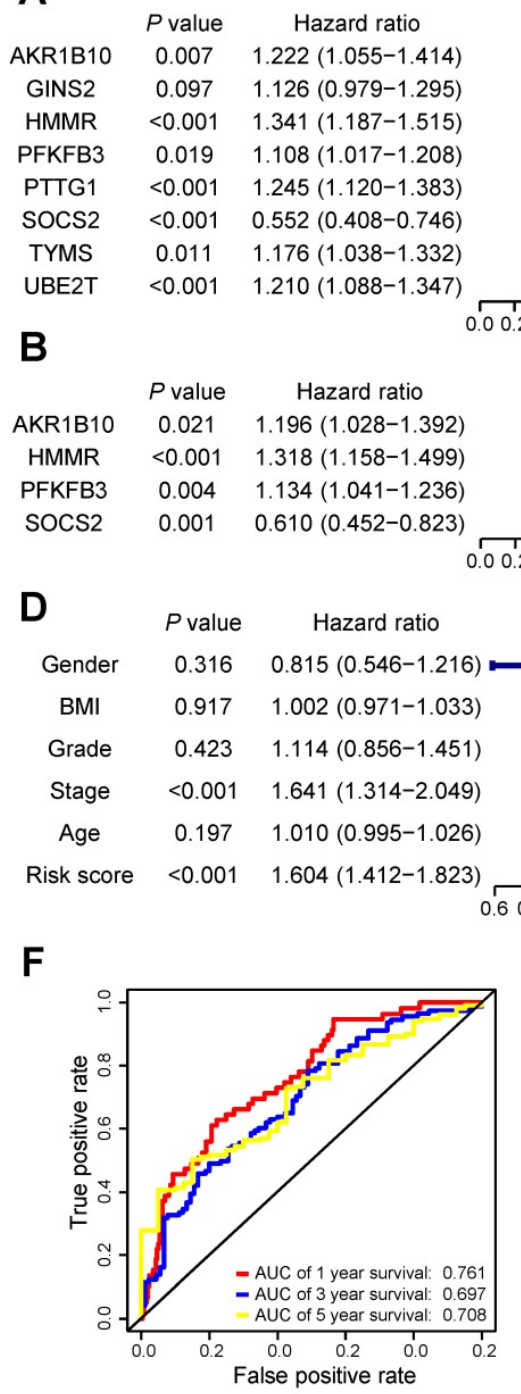

C
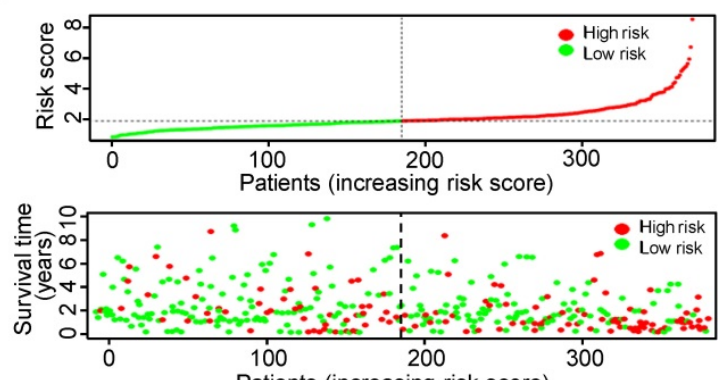

Hazard ratio

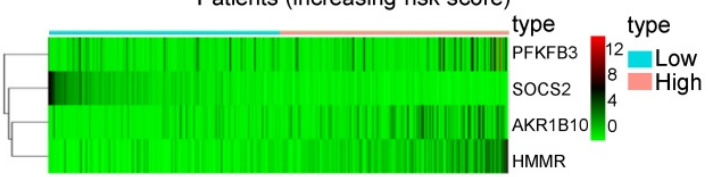

E

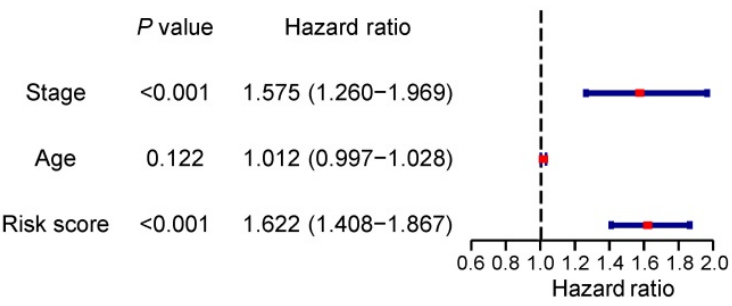

G
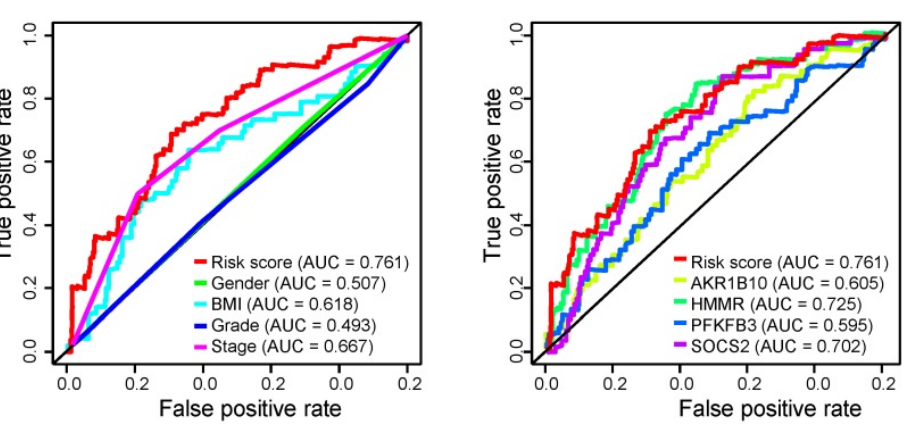

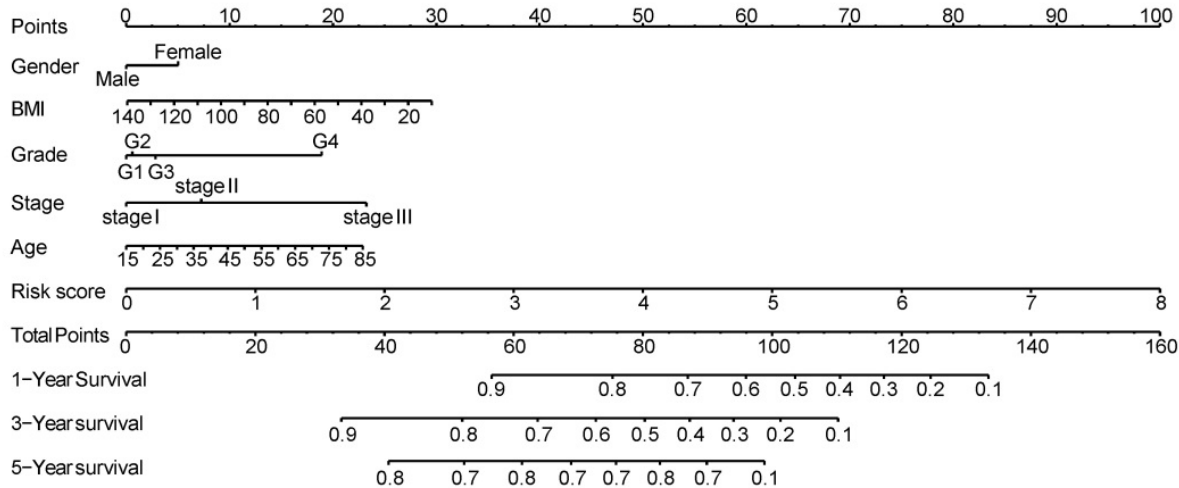

Figure 4. The 4-mRNA prognostic signature and the comprehensive nomogram constructed for HCC patients. (A and $B$ ) The forest plot exhibited genes significantly correlated with overall survival based on univariate (A) and multivariate (B) Cox regression analysis of 22 common DEGs. (C) The distribution of the risk score, survival status and gene expression of the 4-mRNA prognostic signature. (D and $E$ ) The forest plot exhibited prognostic factors significantly correlated with overall survival based on univariate (D) and multivariate (E) Cox regression analysis of risk score and other clinical features. (F) ROC curve was plotted for the prognostic model with 1-, 3-and 5-year overall survival, clinical features, and individual HMMR, AKR1B10, PFKFB3 or SOCS2 in HCC patients. (G) The comprehensive nomogram for 1-, 3-and 5-year overall survival prediction of HCC patients. 
A

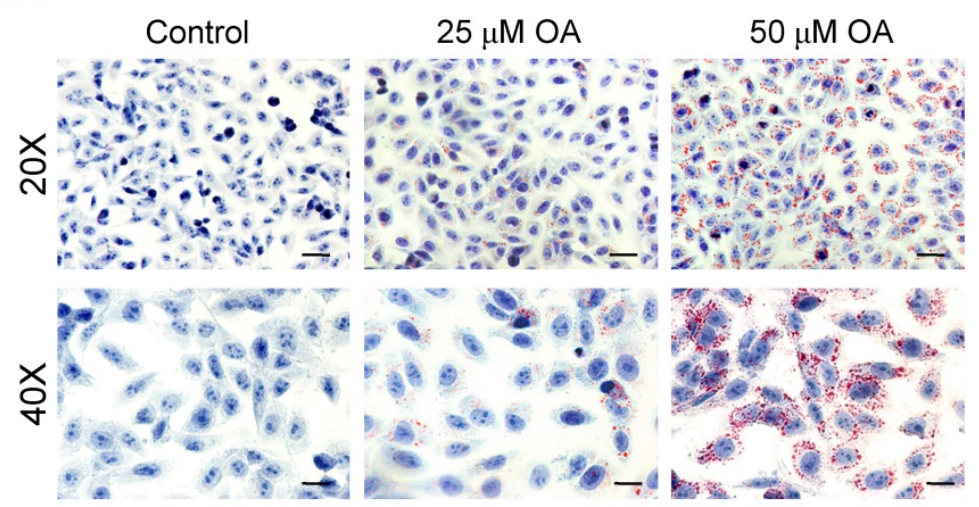

B

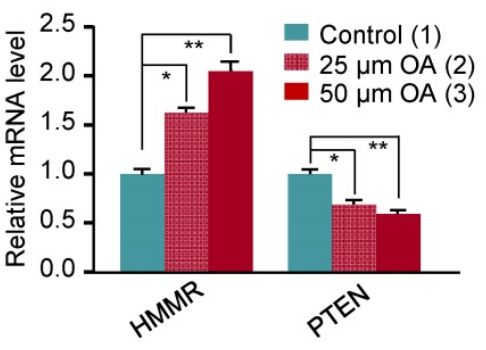

(1) (2)

(3)

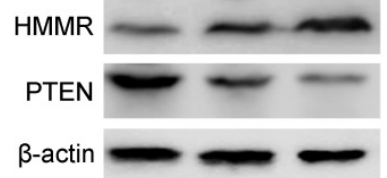

C

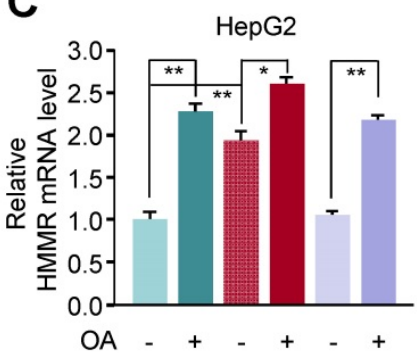

E
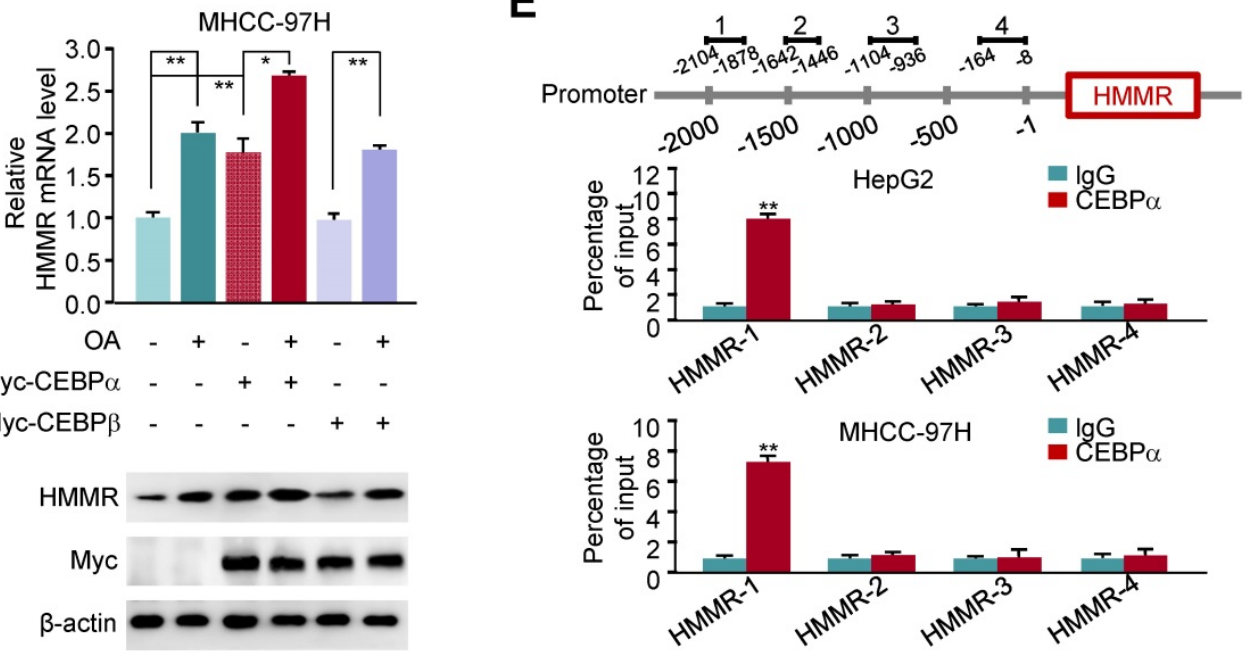

$\mathbf{F}$

D
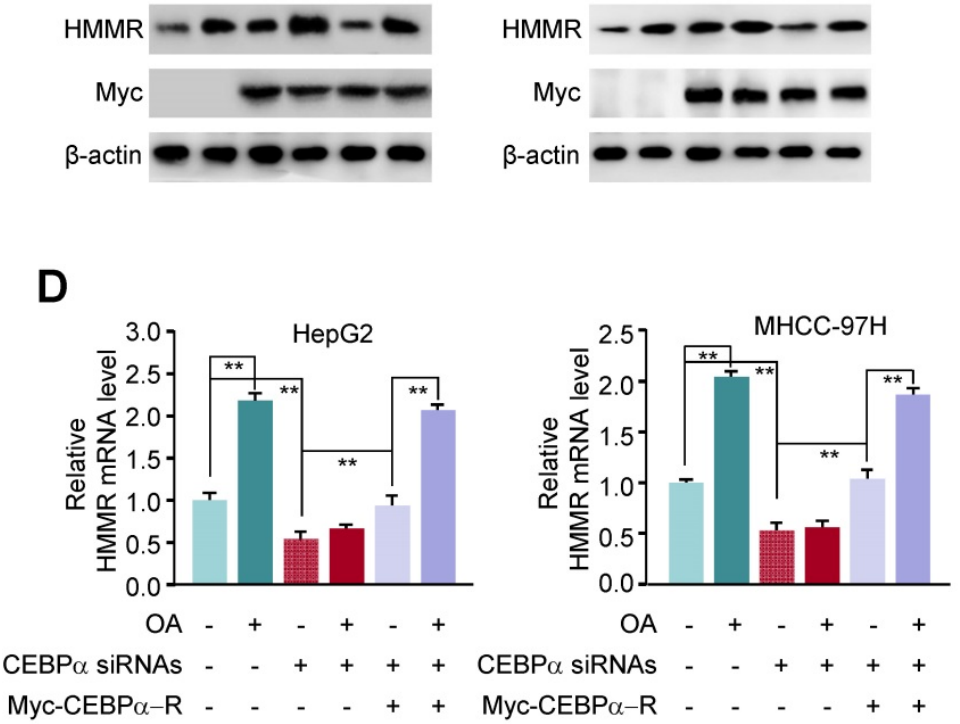

$\alpha$ SiRNAs - -+++
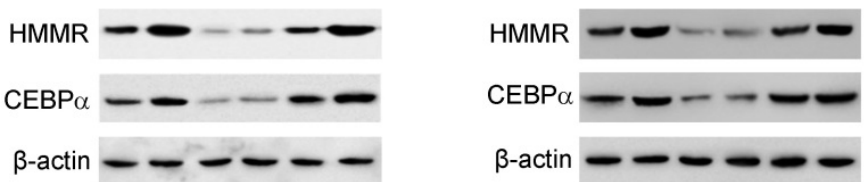
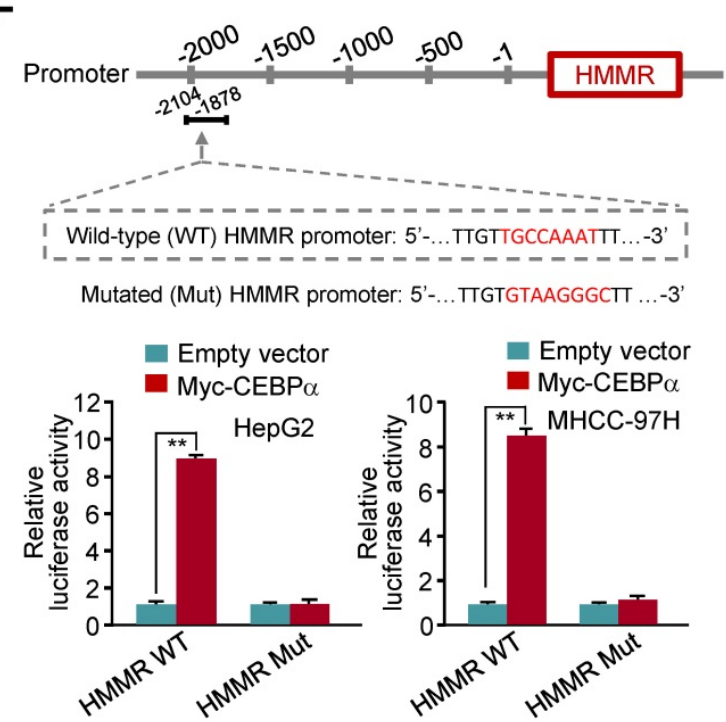

Figure 5. Oleate acid induces HMMR expression via CEBPa. (A) Elevated fat storage in HepG2 cells treated with $25 \mu M$ and $50 \mu M$ oleate acid. Cells were stained with Oil Red O and counterstained with hematoxylin. Scale bar, $50 \mu \mathrm{m}(20 \mathrm{X})$ and $25 \mu \mathrm{m}$ (40X). (B) RT-qPCR and immunoblot analysis of HepG2 cells treated as in (A). RT-qPCR was used for examination of mRNA expression of HMMR and PTEN, and representative immunoblot for their protein expression. $\beta$-actin was used as a loading control. (C) RT-qPCR and immunoblot analysis of HepG2 and MHCC-97H cells transfected with empty vector or Myc-tagged CEBPa or CEBP $\beta$ and treated with $50 \mu M$ oleate acid. (D) RT-qPCR and immunoblot analysis of HepG2 and MHCC-97H cells transfected with control siRNA, CEBPa siRNAs or CEBPa siRNAs plus siRNA-resistant CEBPa plasmid (Myc-CEBPa-R) and treated with $50 \mu \mathrm{M}$ oleate acid. (E) ChIP analysis of CEBPa occupancy on HMMR promoter in HepG2 cells. IgG, normal serum. The different number represents the regions upstream of the transcriptional start site $(-1)$ of HMMR promoter. (F) Luciferase reporter assays of HepG2 and MHCC-97H cells transfected with wild-type or mutated HMMR reporters and Myc-CEBPa. A schematic diagram of the HMMR promoter reporter constructs is shown. Red fonts indicate the putative CEBPa-binding site in human wild-type HMMR promoter. Mutations are introduced into the HMMR promoter. Data shown are mean \pm SD of triplicate measurements that have been repeated 3 times with similar results $(B-F)(* P<0.05$, $* * P<0.01)$. 
A

(1) (2)

FLAG

$\beta$-actin

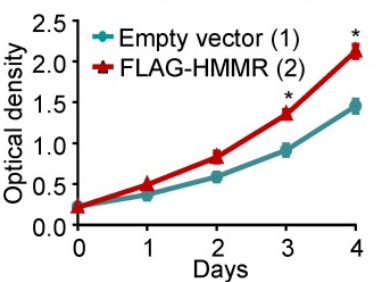

C

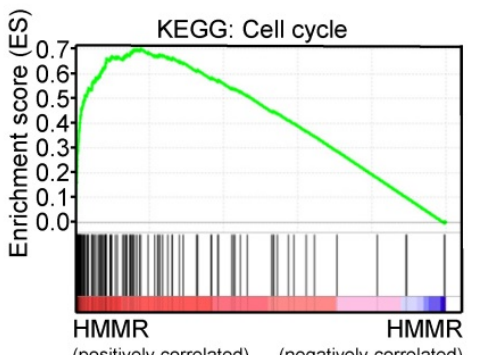

(positively correlated) (negatively correlated) NES:2.155

NOM $P$-val: $<0.001$

FDR $q$-val: 0.001
(1) (2)
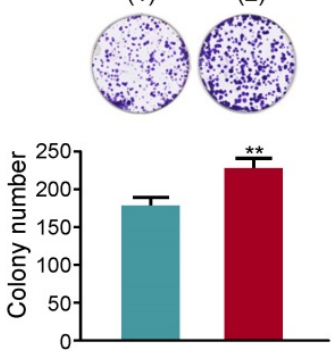

B

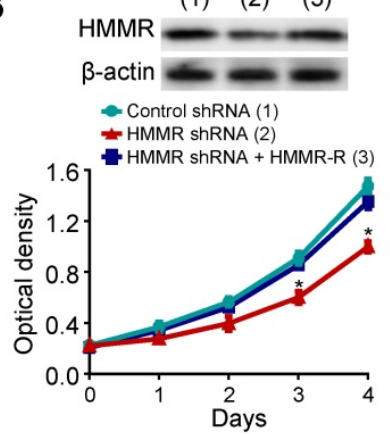

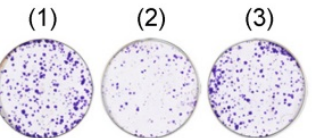

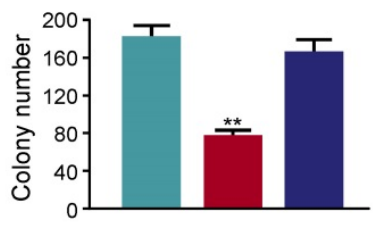

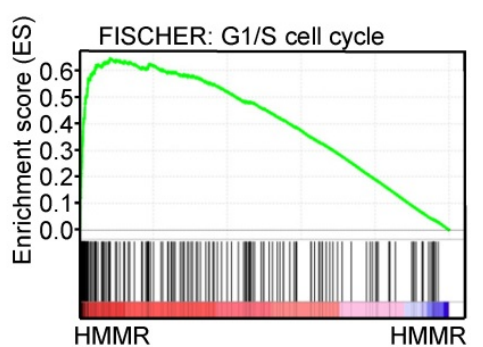

(positively correlated) (negatively correlated) NESitively corre 2182

NOM $P$-val: $<0.00$

FDR $q$-val: 0.002

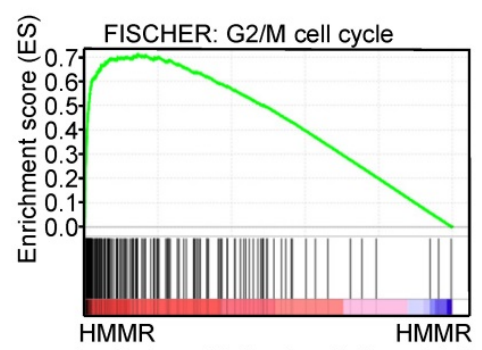

(positively correlated) (negatively correlated) NES: 2.113

NOM $P$-val: $<0.001$

FDR $q$-val: 0.002
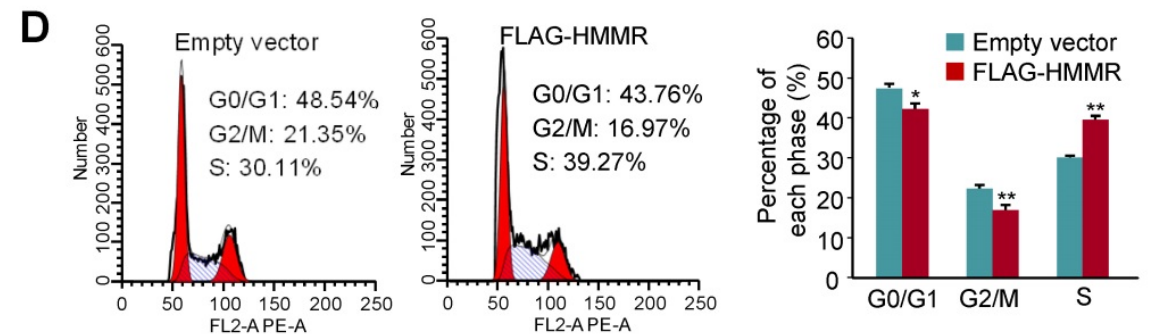

E
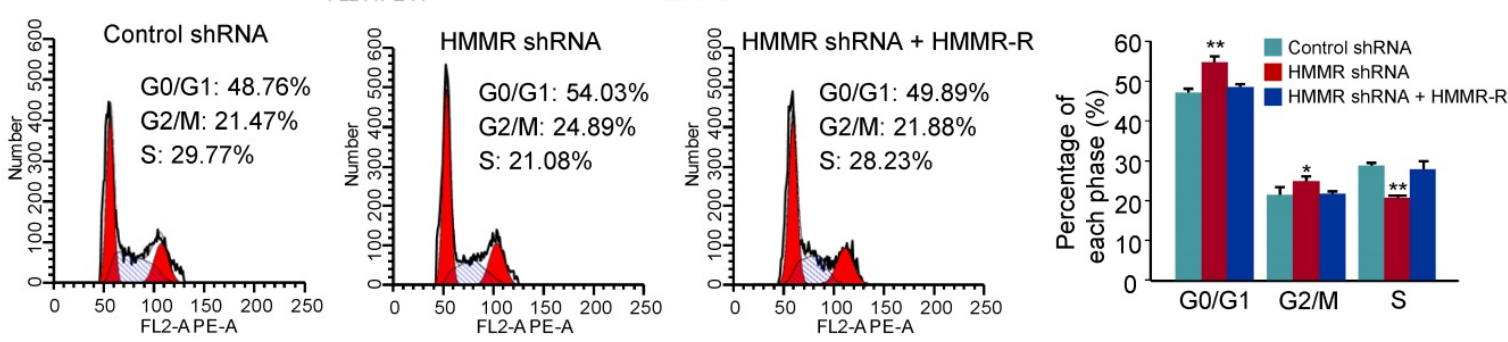

$\mathbf{F}$

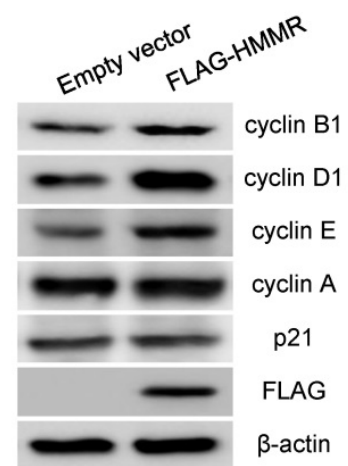

G

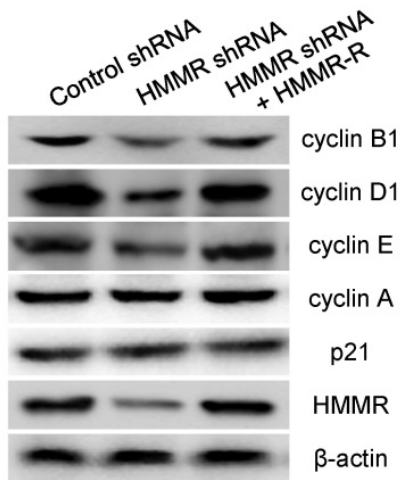

Figure 6. HMMR promotes HepG2 cell proliferation through activating the G1/S and G2/M transitions. (A) HepG2 cells transfected with FLAG-tagged HMMR or empty vector were grown in regular medium and harvested at the indicated times. Cell number and colony formation were detected. Immunoblot showed the expression of FLAG-HMMR. (B) HepG2 cells infected with control shRNA, HMMR shRNA or HMMR shRNA plus siRNA-resistant HMMR (HMMR-R) were cultured and analyzed as in (A). Immunoblot showed HMMR expression. (C) GSEA was conducted to predict the potential mechanism of HMMR promoting HepG2 cell proliferation. (D) Flow cytometry analysis of cell cycle in HepG2 cells transfected with FLAG-HMMR or empty vector. (E) Flow cytometry analysis of cell cycle in HepG2 cells infected with HMMR shRNA or HMMR shRNA plus HMMR-R. The image displayed is one of the representative results. Data shown are mean \pm SD of triplicate measurements that have been repeated 3 times with similar results (A, B, D and E) $(* P<0.05, * * P<0.01$ versus corresponding control). (F) Representative immunoblot of HepG2 cells transfected with FLAG-tagged HMMR or empty vector. (G) Representative immunoblot of HepG2 cells infected with control shRNA, HMMR shRNA or HMMR shRNA plus HMMR-R. 
A
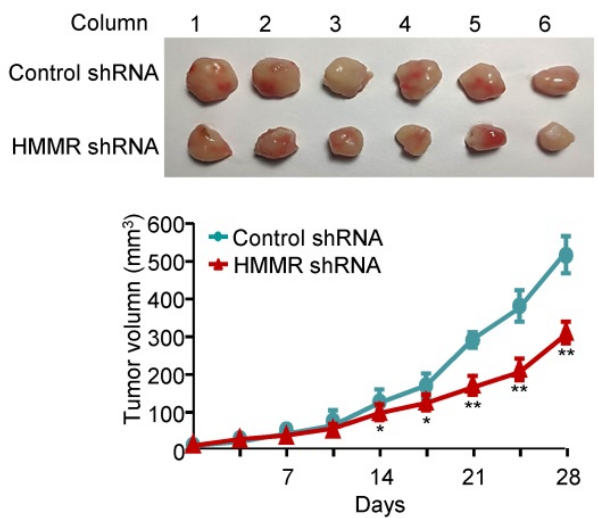

C

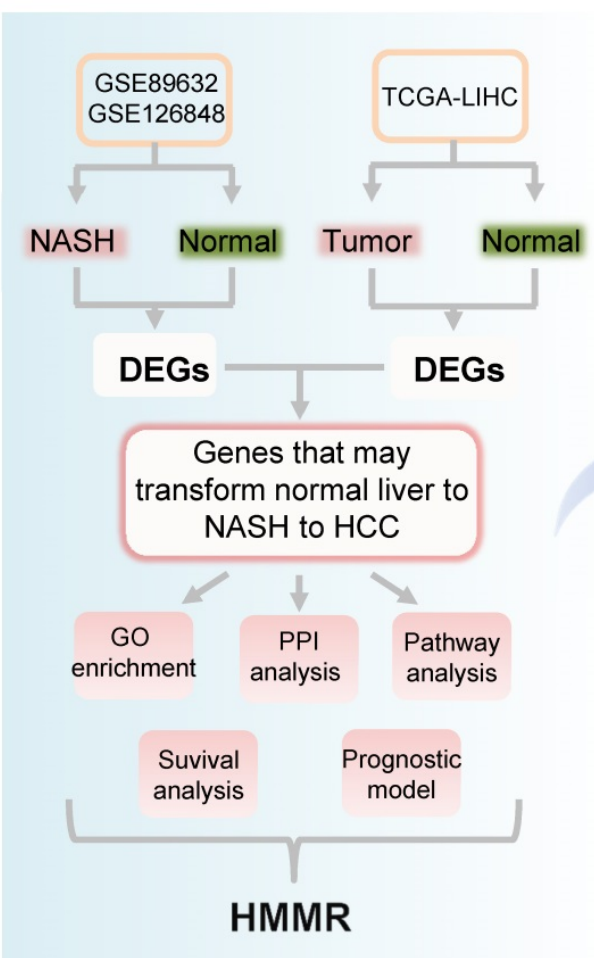

B

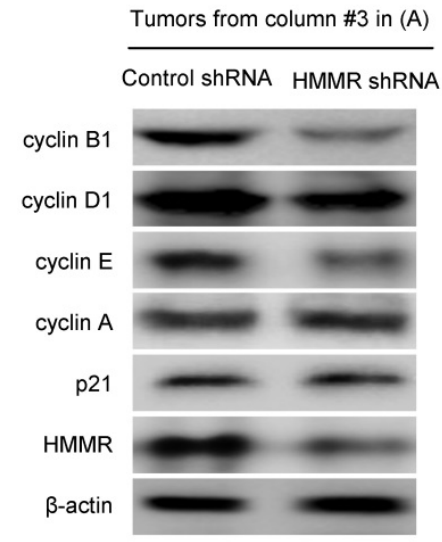


concerned, a HMMR antisense lncRNA, HMMR-AS1, can stabilize HMMR mRNA and promotes cancer progression in lung adenocarcinoma, glioblastoma, and epithelial ovarian cancer [57-59]. We show that OA stimulates the expression of HMMR through transcription factor CEBPa, providing another mechanism underlying HMMR expression.

Hyaluronan (HA), a member of the glycosaminoglycan family, is an extracellular matrix component and interacts with various cellular receptors to promote cell growth and movement [60]. HMMR is one of defined hyaluronan cellular receptors. The evaluation of serum HA could predict liver fibrosis and liver damage in NAFLD patients [61]. HMMR was shown to regulate cancer progression in HA-dependent and -independent manners [52, 62]. Tissue cells and cancer cells with HMMR overexpression tend to be highly proliferative. The exact role of HA/HMMR axismediated NASH/HCC remains to be investigated.

Besides HMMR, the other four hub genes (UBE2T, PTTG1, GINS2 and TYMS) have been shown to play a role in HCC. UBE2T, a member of the E2 family, is demonstrated to be a vital regulator of tumor progression in several cancers, such as lung cancer [63], glioblastoma [64], and HCC [65]. PTTG1 has been reported to be associated with poor prognosis in multiple myeloma [66], prostate cancer [67] and HCC [68]. Overexpression of UBE2T and PTTG1 promotes HCC cell growth, migration and invasion through activating Akt pathway [69, 70]. GINS2 is a member of the GINS complex and participates in DNA replication and cell cycle regulation in most tumors, such as pancreatic cancer [41], bladder cancer [71], and lung cancer [72]. Bioinformatic analysis showed that both individual GINS2 and the whole GINS complex could be prognostic biomarkers for HCC [73]. TYMS is a rate-limiting enzyme in the nucleotide biosynthetic pathway, and involves in DNA synthesis and repair [40]. Interestingly, although upregulated TYMS was associated with poor survival in HCC patients [74], enhanced TYMS activity may protect cancerization of liver tissue by minimizing uracil misincorporation into DNA [75]. However, there are no reports on the relationship between these four hub genes and $\mathrm{NASH}$. More research will be needed to understand the association.

\section{Supplementary Material}

Supplementary figures.

http://www.ijbs.com/v16p2812s1.pdf

Supplementary tables.

http://www.ijbs.com/v16p2812s2.pdf

\section{Acknowledgements}

This work was supported by grants from the National Natural Science Foundation of China (81630067, 81930078 and 81872246).

\section{Competing Interests}

The authors have declared that no competing interest exists.

\section{References}

1. Anstee QM, Reeves HL, Kotsiliti E, Govaere O, Heikenwalder M. From NASH to HCC: current concepts and future challenges. Nat Rev Gastroenterol Hepatol. 2019; 16: 411-28.

2. Rinella ME. Nonalcoholic fatty liver disease: a systematic review. JAMA. 2015; 313: 2263-73.

3. Younossi Z, Anstee QM, Marietti M, Hardy T, Henry L, Eslam M, et al. Global burden of NAFLD and NASH: trends, predictions, risk factors and prevention. Nat Rev Gastroenterol Hepatol. 2018; 15: 11-20.

4. Sanyal A, Poklepovic A, Moyneur E, Barghout V. Population-based risk factors and resource utilization for HCC: US perspective. Curr Med Res Opin. 2010; 26: 2183-91.

5. Jemal A, Bray F, Center MM, Ferlay J, Ward E, Forman D. Global cancer statistics. CA Cancer J Clin. 2011; 61: 69-90.

6. Sircana A, Paschetta E, Saba F, Molinaro F, Musso G. Recent Insight into the Role of Fibrosis in Nonalcoholic Steatohepatitis-Related Hepatocellular Carcinoma. Int J Mol Sci. 2019; 20.

7. Maxwell CA, Keats JJ, Crainie M, Sun X, Yen T, Shibuya E, et al. RHAMM is a centrosomal protein that interacts with dynein and maintains spindle pole stability. Mol Biol Cell. 2003; 14: 2262-76.

8. Zlobec I, Baker K, Terracciano LM, Lugli A. RHAMM, p21 combined phenotype identifies microsatellite instability-high colorectal cancers with a highly adverse prognosis. Clin Cancer Res. 2008; 14: 3798-806.

9. Li H, Guo L, Li JW, Liu N, Qi R, Liu J. Expression of hyaluronan receptors CD44 and RHAMM in stomach cancers: relevance with tumor progression. Int J Oncol. 2000; 17: 927-32.

10. Rein DT, Roehrig K, Schondorf T, Lazar A, Fleisch M, Niederacher D, et al. Expression of the hyaluronan receptor RHAMM in endometrial carcinomas suggests a role in tumour progression and metastasis. J Cancer Res Clin Oncol. 2003; 129: 161-4.

11. Gust KM, Hofer MD, Perner SR, Kim R, Chinnaiyan AM, Varambally S, et al. RHAMM (CD168) is overexpressed at the protein level and may constitute an immunogenic antigen in advanced prostate cancer disease. Neoplasia. 2009; 11: 956-63.

12. Maxwell CA, Rasmussen E, Zhan F, Keats JJ, Adamia S, Strachan E, et al. RHAMM expression and isoform balance predict aggressive disease and poor survival in multiple myeloma. Blood. 2004; 104: 1151-8.

13. Mantripragada KK, Spurlock G, Kluwe L, Chuzhanova N, Ferner RE, Frayling IM, et al. High-resolution DNA copy number profiling of malignant peripheral nerve sheath tumors using targeted microarray-based comparative genomic hybridization. Clin Cancer Res. 2008; 14: 1015-24.

14. Mohan P, Castellsague J, Jiang J, Allen K, Chen H, Nemirovsky O, et al. Genomic imbalance of HMMR/RHAMM regulates the sensitivity and response of malignant peripheral nerve sheath tumour cells to aurora kinase inhibition. Oncotarget. 2013; 4: 80-93.

15. Li H, Frappart L, Moll J, Winkler A, Kroll T, Hamann J, et al. Impaired Planar Germ Cell Division in the Testis, Caused by Dissociation of RHAMM from the Spindle, Results in Hypofertility and Seminoma. Cancer Res. 2016; 76: 6382-95

16. Ouyang G, Yi B, Pan G, Chen X. A robust twelve-gene signature for prognosis prediction of hepatocellular carcinoma. Cancer Cell Int. 2020; 20: 207.

17. Zhou Z, Li Y, Hao H, Wang Y, Zhou Z, Wang Z, et al. Screening Hub Genes as Prognostic Biomarkers of Hepatocellular Carcinoma by Bioinformatics Analysis. Cell Transplant. 2019; 28: 76S-86S.

18. Ni W, Zhang S, Jiang B, Ni R, Xiao M, Lu C, et al. Identification of cancer-related gene network in hepatocellular carcinoma by combined bioinformatic approach and experimental validation. Pathol Res Pract. 2019; 215: 152428

19. Shen S, Kong J, Qiu Y, Yang X, Wang W, Yan L. Identification of core genes and outcomes in hepatocellular carcinoma by bioinformatics analysis. J Cell Biochem. 2019; 120: 10069-81. 
20. Wu HT, Lu FH, Ou HY, Su YC, Hung HC, Wu JS, et al. The role of hepassocin in the development of non-alcoholic fatty liver disease. J Hepatol. 2013; 59: 1065-72.

21. Arendt BM, Comelli EM, Ma DW, Lou W, Teterina A, Kim T, et al. Altered hepatic gene expression in nonalcoholic fatty liver disease is associated with lower hepatic n-3 and n-6 polyunsaturated fatty acids. Hepatology. 2015; 61: 1565-78.

22. Suppli MP, Rigbolt KTG, Veidal SS, Heeboll S, Eriksen PL, Demant M, et al. Hepatic transcriptome signatures in patients with varying degrees of nonalcoholic fatty liver disease compared with healthy normal-weight individuals. Am J Physiol Gastrointest Liver Physiol. 2019; 316: G462-G72.

23. Cerami E, Gao J, Dogrusoz U, Gross BE, Sumer SO, Aksoy BA, et al. The cBio cancer genomics portal: an open platform for exploring multidimensional cancer genomics data. Cancer Discov. 2012; 2: 401-4.

24. Ritchie ME, Phipson B, Wu D, Hu Y, Law CW, Shi W, et al. limma powers differential expression analyses for RNA-sequencing and microarray studies. Nucleic Acids Res. 2015; 43: e47.

25. Guo H, Cai J, Wang X, Wang B, Wang F, Li X, et al. Prognostic values of a novel multi-mRNA signature for predicting relapse of cholangiocarcinoma. Int J Biol Sci. 2020; 16: 869-81.

26. Ling ZA, Xiong DD, Meng RM, Cen JM, Zhao N, Chen G, et al. LncRNA NEAT1 Promotes Deterioration of Hepatocellular Carcinoma Based on In vitro Experiments, Data Mining, and RT-qPCR Analysis. Cell Physiol Biochem. 2018; 48: 540-55.

27. Bader GD, Hogue CW. An automated method for finding molecular complexes in large protein interaction networks. BMC Bioinformatics. 2003; $4: 2$.

28. Chin $\mathrm{CH}$, Chen $\mathrm{SH}, \mathrm{Wu} \mathrm{HH}, \mathrm{Ho} \mathrm{CW}, \mathrm{Ko} \mathrm{MT}$, Lin CY. cytoHubba: identifying hub objects and sub-networks from complex interactome. BMC Syst Biol. 2014; 8 Suppl 4: S11.

29. Shukla S, Evans JR, Malik R, Feng FY, Dhanasekaran SM, Cao X, et al. Development of a RNA-Seq Based Prognostic Signature in Lung Adenocarcinoma. J Natl Cancer Inst. 2017; 109.

30. Aljomah G, Baker SS, Liu W, Kozielski R, Oluwole J, Lupu B, et al. Induction of CYP2E1 in non-alcoholic fatty liver diseases. Exp Mol Pathol. 2015; 99: 677-81.

31. Jeong H, Kim JW, Yang MS, Park C, Kim JH, Lim CW, et al. Beneficial Effects of Korean Red Ginseng in the Progression of Non-Alcoholic Steatohepatitis via FABP4 Modulation. Am J Chin Med. 2018: 1-27.

32. Wang S, Yao Y, Wang X, Zheng G, Ouyang W, Chen W. 25-HC promotes hepatocellular carcinoma metastasis through up-regulation of TLR4 dependent FABP4. Am J Cancer Res. 2019; 9: 2140-55.

33. Han C, Gao L, Zhao L, Sheng Q, Zhang C, An Z, et al. Immunohistochemistry Detects Increased Expression of Aldo-Keto Reductase Family 1 Member B10 (AKR1B10) in Early-Stage Hepatocellular Carcinoma. Med Sci Monit. 2018; 24: 7414-23.

34. Kanno M, Kawaguchi K, Honda M, Horii R, Takatori H, Shimakami T, et al. Serum aldo-keto reductase family 1 member B10 predicts advanced liver fibrosis and fatal complications of nonalcoholic steatohepatitis. J Gastroenterol. 2019; 54: 549-57.

35. Arendt BM, Teterina A, Pettinelli P, Comelli EM, Ma DWL, Fung SK, et al. Cancer-related gene expression is associated with disease severity and modifiable lifestyle factors in non-alcoholic fatty liver disease. Nutrition. 2019; 62: 100-7.

36. Oliva J, Bardag-Gorce F, Lin A, French BA, French SW. The role of cytokines in $\mathrm{UbD}$ promoter regulation and Mallory-Denk body-like aggresomes. Exp Mol Pathol. 2010; 89: 1-8.

37. Skandalis SS, Karalis T, Heldin P. Intracellular hyaluronan: Importance for cellular functions. Semin Cancer Biol. 2020; 62: 20-30.

38. Guo J, Wang M, Wang JP, Wu CX. Ubiquitin-conjugating enzyme E2T knockdown suppresses hepatocellular tumorigenesis via inducing cell cycle arrest and apoptosis. World J Gastroenterol. 2019; 25: 6386-403.

39. $\mathrm{Hu}$ ZG, Zheng $\mathrm{CW}$, Su HZ, Zeng YL, Lin CJ, Guo ZY, et al. MicroRNA-329-mediated PTTG1 downregulation inactivates the MAPK signaling pathway to suppress cell proliferation and tumor growth in cholangiocarcinoma. J Cell Biochem. 2019; 120: 9964-78.

40. Kamb A, Finer-Moore J, Calvert AH, Stroud RM. Structural basis for recognition of polyglutamyl folates by thymidylate synthase. Biochemistry. 1992; 31: 9883-90.

41. Zhang M, He S, Ma X, Ye Y, Wang G, Zhuang J, et al. GINS2 affects cell viability, cell apoptosis, and cell cycle progression of pancreatic cancer cells via MAPK/ERK pathway. J Cancer. 2020; 11: 4662-70.

42. Takeda M, Sakaguchi T, Hiraide T, Shibasaki Y, Morita Y, Kikuchi H, et al. Role of caveolin-1 in hepatocellular carcinoma arising from non-alcoholic fatty liver disease. Cancer Sci. 2018; 109: 2401-11.

43. Park S, Park JH, Jung HJ, Jang JH, Ahn S, Kim Y, et al. A secretome profile indicative of oleate-induced proliferation of HepG2 hepatocellular carcinoma cells. Exp Mol Med. 2018; 50: 93.
44. Kim K, Kim HY, Son EJ, Heo J, Cheong J. Oleic acid inhibits hepatic insulin signaling through deregulation of STAT3 activation and C/EBPalpha expression. Cell Signal. 2009; 21: 1269-76.

45. Brunt EM, Wong VW, Nobili V, Day CP, Sookoian S, Maher JJ, et al. Nonalcoholic fatty liver disease. Nat Rev Dis Primers. 2015; 1: 15080.

46. Wree A, Broderick L, Canbay A, Hoffman HM, Feldstein AE. From NAFLD to NASH to cirrhosis-new insights into disease mechanisms. Nat Rev Gastroenterol Hepatol. 2013; 10: 627-36.

47. Schuster S, Cabrera D, Arrese M, Feldstein AE. Triggering and resolution of inflammation in NASH. Nat Rev Gastroenterol Hepatol. 2018; 15 : 349-64.

48. Piccinin E, Villani G, Moschetta A. Metabolic aspects in NAFLD, NASH and hepatocellular carcinoma: the role of PGC1 coactivators. Nat Rev Gastroenterol Hepatol. 2019; 16: 160-74.

49. Friedman SL, Neuschwander-Tetri BA, Rinella M, Sanyal AJ. Mechanisms of NAFLD development and therapeutic strategies. Nat Med. 2018; 24: 908-22.

50. Connell M, Chen H, Jiang J, Kuan CW, Fotovati A, Chu TL, et al. HMMR acts in the PLK1-dependent spindle positioning pathway and supports neural development. Elife. 2017; 6.

51. Tilghman J, Wu H, Sang $Y$, Shi X, Guerrero-Cazares H, Quinones-Hinojosa A, et al. HMMR maintains the stemness and tumorigenicity of glioblastoma stem-like cells. Cancer Res. 2014; 74: 3168-79.

52. Stevens LE, Cheung WKC, Adua SJ, Arnal-Estape A, Zhao M, Liu Z, et al. Extracellular Matrix Receptor Expression in Subtypes of Lung Adenocarcinoma Potentiates Outgrowth of Micrometastases. Cancer Res. 2017; 77: 1905-17.

53. Rizzardi AE, Vogel RI, Koopmeiners JS, Forster CL, Marston LO, Rosener NK, et al. Elevated hyaluronan and hyaluronan-mediated motility receptor are associated with biochemical failure in patients with intermediate-grade prostate tumors. Cancer. 2014; 120: 1800-9.

54. Spranger S, Jeremias I, Wilde S, Leisegang M, Starck L, Mosetter B, et al. TCR-transgenic lymphocytes specific for HMMR/Rhamm limit tumor outgrowth in vivo. Blood. 2012; 119: 3440-9.

55. Zhang $\mathrm{H}$, Ren $\mathrm{L}$, Ding $\mathrm{Y}, \mathrm{Li}$ F, Chen $\mathrm{X}$, Ouyang $\mathrm{Y}$, et al. Hyaluronan-mediated motility receptor confers resistance to chemotherapy via TGFbeta/Smad2-induced epithelial-mesenchymal transition in gastric cancer. FASEB J. 2019; 33: 6365-77.

56. Zlobec I, Terracciano L, Tornillo L, Gunthert U, Vuong T, Jass JR, et al. Role of RHAMM within the hierarchy of well-established prognostic factors in colorectal cancer. Gut. 2008; 57: 1413-9.

57. Cai Y, Sheng Z, Chen Y, Wang J. LncRNA HMMR-AS1 promotes proliferation and metastasis of lung adenocarcinoma by regulating MiR-138/sirt6 axis. Aging (Albany NY). 2019; 11: 3041-54.

58. Li J, Ji X, Wang H. Targeting Long Noncoding RNA HMMR-AS1 Suppresses and Radiosensitizes Glioblastoma. Neoplasia. 2018; 20: 456-66.

59. Chu ZP, Dai J, Jia LG, Li J, Zhang Y, Zhang ZY, et al. Increased expression of long noncoding RNA HMMR-AS1 in epithelial ovarian cancer: an independent prognostic factor. Eur Rev Med Pharmacol Sci. 2018; 22: 8145-50.

60. Spinelli FM, Vitale DL, Sevic I, Alaniz L. Hyaluronan in the Tumor Microenvironment. Adv Exp Med Biol. 2020; 1245: 67-83.

61. Valenti L, Dongiovanni P. Serum hyaluronic acid for the screening of progressive nonalcoholic steatohepatitis in children: a promising approach. Transl Res. 2010; 156: 226-8.

62. He Z, Mei L, Connell M, Maxwell CA. Hyaluronan Mediated Motility Receptor (HMMR) Encodes an Evolutionarily Conserved Homeostasis, Mitosis, and Meiosis Regulator Rather than a Hyaluronan Receptor. Cells. 2020; 9.

63. Yin $\mathrm{H}$, Wang $\mathrm{X}$, Zhang $\mathrm{X}$, Zeng $\mathrm{Y}, \mathrm{Xu} \mathrm{Q}$, Wang $\mathrm{W}$, et al. UBE2T promotes radiation resistance in non-small cell lung cancer via inducing epithelial-mesenchymal transition and the ubiquitination-mediated FOXO1 degradation. Cancer Lett. 2020.

64. Huang P, Guo Y, Zhao Z, Ning W, Wang H, Gu C, et al. UBE2T promotes glioblastoma invasion and migration via stabilizing GRP78 and regulating EMT. Aging (Albany NY). 2020; 12: 10275-89.

65. Wei X, You X, Zhang J, Zhou C. MicroRNA-1305 Inhibits the Stemness of LCSCs and Tumorigenesis by Repressing the UBE2T-Dependent Akt-Signaling Pathway. Mol Ther Nucleic Acids. 2019; 16: 721-32.

66. Noll JE, Vandyke K, Hewett DR, Mrozik KM, Bala RJ, Williams SA, et al. PTTG1 expression is associated with hyperproliferative disease and poor prognosis in multiple myeloma. J Hematol Oncol. 2015; 8: 106.

67. Ersvaer E, Kildal W, Vlatkovic L, Cyll K, Pradhan M, Kleppe A, et al. Prognostic value of mitotic checkpoint protein BUB3, cyclin B1, and pituitary tumor-transforming 1 expression in prostate cancer. Mod Pathol. 2020; 33: 905-15. 
68. Fujii T, Nomoto S, Koshikawa K, Yatabe Y, Teshigawara O, Mori T, et al. Overexpression of pituitary tumor transforming gene 1 in HCC is associated with angiogenesis and poor prognosis. Hepatology. 2006; 43: 1267-75.

69. Tao Y, Li R, Shen C, Li J, Zhang Q, Ma Z, et al. SENP1 is a crucial promotor for hepatocellular carcinoma through deSUMOylation of UBE2T. Aging (Albany NY). 2020; 12: 1563-76.

70. Huang JL, Cao SW, Ou QS, Yang B, Zheng SH, Tang J, et al. The long non-coding RNA PTTG3P promotes cell growth and metastasis via up-regulating PTTG1 and activating PI3K/AKT signaling in hepatocellular carcinoma. Mol Cancer. 2018; 17: 93.

71. Dai G, Huang C, Yang J, Jin L, Fu K, Yuan F, et al. LncRNA SNHG3 promotes bladder cancer proliferation and metastasis through miR-515-5p/GINS2 axis. J Cell Mol Med. 2020.

72. Liu $X$, Sun L, Zhang $S$, Zhang $S$, Li W. GINS2 facilitates epithelial-to-mesenchymal transition in non-small-cell lung cancer through modulating PI3K/Akt and MEK/ERK signaling. J Cell Physiol. 2019.

73. Lian YF, Li SS, Huang YL, Wei H, Chen DM, Wang JL, et al. Up-regulated and interrelated expressions of GINS subunits predict poor prognosis in hepatocellular carcinoma. Biosci Rep. 2018; 38.

74. Yeh HW, Lee SS, Chang CY, Hu CM, Jou YS. Pyrimidine metabolic rate limiting enzymes in poorly-differentiated hepatocellular carcinoma are signature genes of cancer stemness and associated with poor prognosis. Oncotarget. 2017; 8: 77734-51.

75. Studach LL, Menne S, Cairo S, Buendia MA, Hullinger RL, Lefrancois L, et al. Subset of Suz12/PRC2 target genes is activated during hepatitis B virus replication and liver carcinogenesis associated with HBV X protein. Hepatology. 2012; 56: 1240-51. 